September 2009

\title{
Decompactifications and Massless D-Branes in Hybrid Models
}

\author{
Paul S. Aspinwall and M. Ronen Plesser \\ Center for Geometry and Theoretical Physics, Box 90318 \\ Duke University, Durham, NC 27708-0318
}

\begin{abstract}
A method of determining the mass spectrum of BPS D-branes in any phase limit of a gauged linear sigma model is introduced. A ring associated to monodromy is defined and one considers K-theory to be a module over this ring. A simple but interesting class of hybrid models with Landau-Ginzburg fibres over $\mathbb{P}^{n}$ are analyzed using special Kähler geometry and D-brane probes. In some cases the hybrid limit is an infinite distance in moduli space and corresponds to a decompactification. In other cases the hybrid limit is at a finite distance and acquires massless D-branes. An example studied appears to correspond to a novel theory of supergravity with an $\mathrm{SU}(2)$ gauge symmetry where the gauge and gravitational couplings are necessarily tied to each other.
\end{abstract}




\section{Introduction}

A huge class of $N=(2,2)$ superconformal field theories are associated with the gauged linear $\sigma$-model of Witten [1]. This is an asymptotically free gauge theory which flows at low energies to a nontrivial superconformal field theory. The parameters of the gauged linear $\sigma$-model include the coefficients $r$ of Fayet-Iliopoulos $D$-terms, and for large $|r|$ the gauge symmetry is broken at high energies, where a perturbative treatment of the gauge interaction is valid. It is in these asymptotic regions that the gauged linear $\sigma$-model is a reliable guide to the low-energy physics. As is well-known, this model exhibits a "phase" structure, dividing $r$-space into cones. In each of these the space of classical vacua is naturally modeled by a specific toric geometry; this description is expected to be reliable deep in the interior of each cone. The significance of the limit points is especially clear in the topological twisted version of the theory. Here each limit point is the center of an instanton expansion. The most familiar examples are Calabi-Yau phases and Landau-Ginzburg limit points.

If one considers an example where the moduli space has more than a few dimensions, there will be hundreds of phases and most of these phases will not have a simple interpretation as a Calabi-Yau space (with partially resolved orbifolds) or a Landau-Ginzburg theory. In a typical phase the space of classical vacua will be reducible, including components of different dimensions. In some of these vacua there will be massless fields in addition to the coordinates on the space of vacua. These interact via an effective superpotential. Each irreducible component can naïvely be thought of as a fibration over some geometric space where the fibre is a Landau-Ginzburg theory and these phases are known as "hybrid" phases $[1,2]$. Despite their ubiquity, hybrid phases have not been studied as thoroughly as the types of phases mentioned above.

The purpose of this paper is to explore the geometry of a few simple hybrid phases by using D-brane probes to see how accurate the above fibration picture is. In particular we want to study the geometry in the "limit" of such hybrid phases, deep in the interior of the associated cone. In this limit the gauge symmetry is broken at high energy and a classical calculation shows that the base of the fibration (the space of classical vacua) grows large, meaning the massless fields corresponding to motion along the base appear weakly coupled.

We find two types of behavior in the hybrid limit. In hybrid phases of the first type, which we will call true hybrid phases, the base of the fibration indeed grows large, and the description of the theory as a Landau-Ginzburg model fibered over the base is valid. The hybrid limit point, corresponding to a decompactification of the target space, is at an infinite distance (in the Zamolodchikov metric) in moduli space. The gauge instanton expansion about this limit is an expansion in worldsheet instantons wrapping holomorphic curves in the base space.

In hybrid phases of the second type, which we call pseudo-hybrid phases, the description of the low-energy limit as a fibration is not valid. The base space does not grow large, but remains at a finite size in the limit. These limit points are at a finite distance in the moduli space, and instead of a weakly-coupled decompactification limit we find that they describe singular conformal field theories. The singularity is associated in string theory to the appearance of massless D-branes at the hybrid limit. The spacetime physics near such 
a singularity is an interesting strongly-interacting theory with some unfamiliar properties.

A central mathematical problem in this paper is the determination of the asymptotic form of D-brane masses as we go into the hybrid limit. This can be solved by directly solving the associated Picard-Fuch's differential equations from mirror symmetry. However, rather than doing this we find it much more enlightening to introduce a construction we call the "monodromy ring". The ring arises from an observation in [3] that the monodromy on D-branes around phase limits has a particularly simple form when the category of D-branes is written in terms of specific tilting collections. By using the monodromy ring we can, with very little effort, determine the asymptotic form of the relevant periods in phase limits.

The monodromy ring results also allow us to easily construct candidates for particular D-branes with a corresponding class associated to a given period. By doing this we can find candidate "0-branes". That is, D-branes naturally associated with points in the hybrid geometry. Such 0-branes are not at all the same D-branes that would be called 0-branes in the large radius limit. These 0 -branes allow us to probe the geometry of the hybrid phases. A defining property of these 0-branes is that they are light, i.e., lighter than 2-branes, 4-branes, etc., as one approaches the phase limit.

The geometry of hybrid models has been studied elsewhere, in [4,5], for example. The work of [5] using twisted derived categories seems important for the limits associated to a decompactification but uses a somewhat different perspective than we adopt in this paper.

In section 2 we describe the mathematical machinery of the monodromy ring. Section 3 discusses the two simplest cases of the hybrid model and we see how they correspond to decompactifications in, respectively, one and three complex dimensions. In section 4 we discuss another very simple hybrid model but discover that this does not correspond to a decompactification but, rather, a curious model of $N=2$ supergravity with an $\mathrm{SU}(2)$ gauge symmetry that is somehow unavoidably intertwined with gravity.

\section{Monodromy}

We will need to compute the asymptotic form of periods for D-branes near limit points in the moduli space of gauged linear $\sigma$-models. To do this we use the monodromy ring defined below. The mathematics of computing periods is well-established beginning with the work of [6] and consists of solving Picard-Fuchs equations, say in the large radius phase, and analytically continuing into the other phases. Here we develop a somewhat easier method which should become particularly useful when applied to cases of more than one modulus. In this paper we consider only moduli spaces of dimension one and the periods can be obtained fairly painlessly without resorting to the monodromy ring. Having said that, the monodromy ring analysis lends itself well to finding specific D-branes for our candidate 0-branes rather than just D-brane charges. 


\subsection{Noncompact Calabi-Yaus}

While the main object of interest in this paper is a compact Calabi-Yau threefold we will first discuss the simpler case where the Calabi-Yau is toric and noncompact. We refer to [7] for more details of some of the constructions below.

Assume we have a noncompact toric Calabi-Yau threefold $X$. This has a homogeneous coordinate ring [8] $S=\mathbb{C}\left[x_{1}, x_{2}, x_{3}, \ldots, x_{n}\right]$. There is a fan $\Sigma$ and each homogeneous coordinate $x_{i}$ is associated with one of the one-dimensional rays of $\Sigma$. The Cox ideal in $S$ is defined as

$$
B_{\Sigma}=\left(\prod_{i \notin \sigma_{1}} x_{i}, \prod_{i \notin \sigma_{2}} x_{i}, \ldots\right),
$$

where $i \notin \sigma$ means that the ray $i$ is not in a cone $\sigma$ of the fan $\Sigma$. We also have an $r \times n$ charge matrix $Q$ which defines a $\left(\mathbb{C}^{*}\right)^{r}$ torus action:

$$
x_{i} \mapsto \lambda_{1}^{Q_{1 i}} \lambda_{2}^{Q_{2 i}} \ldots \lambda_{r}^{Q_{r i}} x_{i}
$$

where $\lambda_{j} \in \mathbb{C}^{*}$. This gives $S$ the structure of an $r$-graded ring. The toric variety $X$ is then defined as the quotient $\left(\mathbb{C}^{n}-V\left(B_{\Sigma}\right)\right) /\left(\mathbb{C}^{*}\right)^{r}$, where $V\left(B_{\Sigma}\right)$ is the collection of points where all elements of the ideal $B_{\Sigma}$ vanish.

The category of B-type D-branes on $X$ is then given $[3,7,8]$ by the quotient:

$$
\mathbf{D}(X)=\frac{\mathbf{D}(\mathrm{gr}-S)}{T_{\Sigma}},
$$

where $\mathbf{D}(\operatorname{gr}-S)$ is the bounded derived category of finitely generated graded $S$-modules and $T_{\Sigma}$ is the full subcategory of modules killed by some power of the Cox ideal $B_{\Sigma}$. This quotient of triangulated categories is as in [9].

The associated gauged linear $\sigma$-model has chiral multiplets $\Phi_{1}, \Phi_{2}, \Phi_{3}, \ldots, \Phi_{n}$ interacting with $r$ gauge multiplets. The gauge group acts as in (2) with $|\lambda|=1$. $X$ is Calabi-Yau if the charges satisfy

$$
\sum_{i} Q_{i}=0
$$

and this ensures the existence of a non-anomalous $R$-symmetry in the theory which descends to the $\mathrm{U}(1)_{R}$ of the low-energy superconformal field theory 1 There are $r$ Fayet-Iliopoulos couplings and a cone in $\mathbb{R}^{r}$ such that for values of the couplings in this cone, the $D$-term conditions constrain the expectation values of the scalars $x_{i}$ in the chiral multiplets to lie away from $V\left(B_{\Sigma}\right)$. The space of gauge-inequivalent vacua is then precisely $X$.

In all of our examples here $r=1$ and there will be one coupling conventionally denoted $r$. We choose the charges so that the Calabi-Yau cone is given by $r>0$. The fields $x_{i}$ with negative charge are relabeled $p_{a}$. For these values of $r$ the space of gauge inequivalent vacua

\footnotetext{
${ }^{1}$ The noncompact field space means this theory requires boundary conditions to be specified for the fields. In practice, we consider this theory either as a local description near a singularity in a compact Calabi-Yau or as a step in constructing a compact model as in section 2.2 .
} 
is the total space $X$ of a smooth bundle over a compact toric variety for which $x_{i}$ generate the homogeneous coordinate ring. For $r<0$ the space of vacua will typically be a $V$-bundle over a toric variety for which $p_{a}$ generate the homogeneous coordinate ring.

\subsubsection{The monodromy ring}

An interesting observation in [3] is that the monodromy of D-branes around the linear $\sigma$ model limit associated to $\Sigma$ has a very simple description in terms of (3). It amounts to a simple shift in the grading.

Let us introduce a construction for the associated K-theory of the D-branes. Consider first the K-theory of the category $\mathbf{D}(\mathrm{gr}-S)$. This is the free abelian group generated all objects in $\mathbf{D}(\mathrm{gr}-S)$ divided out by the relation $[\mathrm{a}]-[\mathrm{b}]+[\mathrm{c}]=0$ for any distinguished triangle

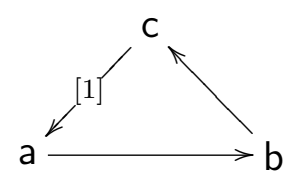

Any object in $\mathbf{D}(\mathrm{gr}-S)$ has a finite resolution in terms of the shifted modules $S(\mathbf{v})$ where $\mathbf{v}$ is an $r$-component vector of integers. There are no nontrivial triangles with entries purely of the form $S(\mathbf{v})$ and so the K-theory group, $K(\operatorname{gr}-S)$, of $\mathbf{D}(\mathrm{gr}-S)$ is isomorphic to the abelian group of bounded Laurent polynomials in $r$ variables. That is, we have an isomorphism

$$
k: K(\mathrm{gr}-S) \stackrel{\simeq}{\longrightarrow} \mathbb{Z}\left[s_{1}, s_{1}^{-1}, s_{2}, s_{2}^{-1}, \ldots, s_{r}, s_{r}^{-1}\right],
$$

where $k(S(\mathbf{v}))=s_{1}^{v_{1}} s_{2}^{v_{2}} \cdots s_{r}^{v_{r}}$.

The K-theory of the derived category $\mathbf{D}(X)$ is the usual topological K-theory $K(X)$ if $X$ is a manifold (and we may use this as the definition of $K(X)$ if $X$ is not a manifold) [10].

In the quotient (3), the K-theory of the subcategory $T_{\Sigma}$ will correspond to a subgroup of $\mathbb{Z}\left[s_{1}, s_{1}^{-1}, s_{2}, s_{2}^{-1}, \ldots, s_{r}, s_{r}^{-1}\right]$. Since $T_{\Sigma}$ is closed under shifting the grading, this subgroup will actually be an ideal. We therefore have

$$
K(X)=\frac{\mathbb{Z}\left[s_{1}, s_{1}^{-1}, s_{2}, s_{2}^{-1}, \ldots, s_{r}, s_{r}^{-1}\right]}{k\left(T_{\Sigma}\right)} .
$$

We may locally view a punctured neighborhood of any limit point in the moduli space as $\left(\mathbb{C}^{*}\right)^{r}$. According to [3], the monodromy around this limit point within the $i$ th copy of $\mathbb{C}^{*}$ may be obtained by shifting the $i$ th grading by one. That is, in terms of (7), such monodromy amounts to multiplication by $s_{i}$. This puts a ring structure on $K(X)$. We will refer to this as the monodromy ring.

In the large radius limit monodromy amounts to tensoring by a line bundle $\mathscr{L}$. In $\mathrm{K}$ theory this corresponds to a wedge product by the Chern character $\operatorname{ch}(\mathscr{L})$. This gives a direct link in the large radius phase between the monodromy ring and the cohomology ring. Such a link is less clear in the other phases. 


\subsubsection{An example $-\mathbb{C}^{3} / \mathbb{Z}_{3}$}

All of this is most easily understood in the context of an example so let us consider the case of the blow-up of $\mathbb{C}^{3} / \mathbb{Z}_{3}$. Here we use the notation

$$
S=\mathbb{C}\left[p, x_{1}, x_{2}, x_{3}\right]
$$

and the charges are $(-3,1,1,1)$ respectively.

In the orbifold phase $\Sigma$ consists of a single cone and $B_{\Sigma}=(p)$. Thus, in the D-brane category $\mathbf{D}(X)$, the module $S /(p)$ is trivial. This module is quasi-isomorphic to the complex

$$
S(3) \stackrel{p}{\longrightarrow} S \text {. }
$$

This means that the map $p$ above is an isomorphism in the quotient category $\mathbf{D}(X)$ given by (3). Similarly, since $S(m) /(p)$ is trivial for any $m$ we have isomorphisms of D-branes

$$
S(m) \cong S(m+3)
$$

in the orbifold phase.

Monodromy around the limit point, i.e., the orbifold point, is simply given by shifting the grading by one. Thus the equivalence (10) shows immediately that there is a $\mathbb{Z}_{3}$ monodromy around the orbifold point in moduli space.

The other phase is the large radius limit phase where $B_{\Sigma}=\left(x_{1}, x_{2}, x_{3}\right)$. Here the monodromy is not finite. To understand the monodromy we can look at D-brane charges or, equivalently, K-theory. That is, we look at the monodromy ring. We have a resolution

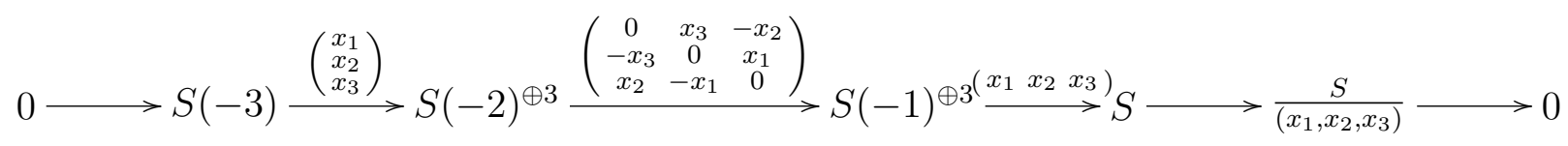

The ideal $k\left(T_{\Sigma}\right)$ must therefore contain

$$
1-3 s^{-1}+3 s^{-2}-s^{-3}=\left(1-s^{-1}\right)^{3} .
$$

In fact the ideal $k\left(T_{\Sigma}\right)$ is generated by this. We denote this principal ideal as $\left(\left(1-s^{-1}\right)^{3}\right)$, which is equivalent to $\left((s-1)^{3}\right)$, since $s$ is a unit. Thus the monodromy ring is

$$
K(X)=\frac{\mathbb{Z}\left[s, s^{-1}\right]}{\left((s-1)^{3}\right)} .
$$

This makes it clear that the monodromy action on D-brane charges is unipotent of order 3 , i.e., it obeys $(s-1)^{3}=0$.

Clearly in the orbifold phase we have a monodromy ring

$$
K(X)=\frac{\mathbb{Z}\left[s, s^{-1}\right]}{\left(s^{3}-1\right)} .
$$

It is worth noting that we are discussing the K-theory finitely generated by vector bundles on $X$. This means that we actually miss the D-brane charge of a 0-brane. Writing a free resolution of a skyscraper sheaf, $\mathscr{O}_{x}$, associated to a point in $X$ one can show that $k\left(\mathscr{O}_{x}\right)=0$. This problem is caused by the fact that $X$ is noncompact. Fortunately the K-theory captures all of the D-branes charges once we consider compact examples. 


\subsection{Compact Calabi-Yaus}

\subsubsection{The category of D-branes}

Our main interest is in compact Calabi-Yau spaces. To construct a gauged linear $\sigma$-model whose low-energy physics is associated to a compact Calabi-Yau spaces [1] we introduce into the model described above a superpotential interaction determined by a gauge-invariant polynomial in the fields which we assume is of the form

$$
\mathcal{W}=\sum_{a} p_{a} f_{a}(x)
$$

where $f_{a}$ are homogeneous polynomials in $x_{i}$ such that $\mathcal{W}$ is gauge invariant. The low-energy dynamics in the large- $r$ limit is that of a nonlinear $\sigma$-model on the critical point set of $\mathcal{W}$. For sufficiently generic $f_{a}$ this is the intersection of all the hypersurfaces $f_{a}=0$ and $p_{a}=0$ in $V$. The condition (4) together with the fact that all the $x_{i}$ 's have positive charge ensure that $X$ is a compact Calabi-Yau variety. The $\mathrm{U}(1)_{R}$ symmetry is broken by the superpotential, but combining this with the global (non- $R$ ) symmetry assigning charge 2 to $p_{a}$ and charge 0 to $x_{i}$, under which $\mathcal{W}$ has charge 2 , produces an unbroken symmetry which descends again to the $\mathrm{U}(1)_{R}$ symmetry of the superconformal field theory.

Following our discussion of the noncompact case, let $S$ denote the homogeneous coordinate ring of $V . S$ has an $r$-fold grading from the toric data as at the start of this section. In addition we add one further grading associated to the $R$-symmetry. This grading lives in $2 \mathbb{Z}$, i.e., it is always an even number.

Define the category $\operatorname{DGr} S(\mathcal{W})$ of matrix factorizations of $\mathcal{W}$ as follows. An object is a pair

$$
\bar{P}=\left(P_{1} \underset{u_{0}}{\stackrel{u_{1}}{\rightleftarrows}} P_{0}\right),
$$

where $P_{0}$ and $P_{1}$ are two finite rank graded free $S$-modules. The two maps satisfy the matrix factorization condition

$$
u_{0} u_{1}=u_{1} u_{0}=\mathcal{W} .1,
$$

and $u_{0}$ is a map of degree 2 with respect to the $R$-symmetry while $u_{1}$ has degree 0 . Both $u_{0}$ and $u_{1}$ have degree 0 with respect to the toric gradings. Morphisms are defined in the obvious way up to homotopy. We refer to $[11,12]$ for more details. The category $\operatorname{DGr} S(\mathcal{W})$ is a triangulated category with a shift functor

$$
\bar{P}[1]=\left(P_{0} \underset{u_{1}}{\stackrel{u_{0}}{\rightleftarrows}} P_{1}\{2\}\right),
$$

where \{\} denotes a shift in the $R$-grading. Thus

$$
\bar{P}[2]=\bar{P}\{2\},
$$

and the $R$-symmetry grading is identified with the homological grading (and extended from $2 \mathbb{Z}$-valued to $\mathbb{Z}$-valued). That is, there is no difference between $[m]$ and $\{m\}$ in this category although we will sometimes use both notations for clarity. 
The category $\operatorname{DGr} S(\mathcal{W})$ should be considered to be the category of D-branes before the conditions imposed by the $D$-terms of the gauged linear $\sigma$-model have been imposed. It is the analogue of $\mathbf{D}(\mathrm{gr}-S)$ for the noncompact Calabi-Yau case considered above.

There is an equivalence of categories [11]

$$
\operatorname{DGr} S(\mathcal{W}) \cong \frac{\mathbf{D}\left(\operatorname{gr}-S^{\prime}\right)}{\mathfrak{P e r f}\left(S^{\prime}\right)},
$$

where $S^{\prime}=S /(\mathcal{W})$ and $\mathfrak{P e r f}\left(S^{\prime}\right)$ denotes the subcategory of "perfect complexes", i.e., finite length complexes of finitely-generated modules. This equivalence is made manifest by an observation by Eisenbud [13]. Any free $S$-resolution of an $S$-module annihilated by $\mathcal{W}$ will ultimately becomes periodic of length 2 as one moves sufficiently far left in the resolution. This asymptotic form of the resolution can be written as a matrix factorization (17).

The actual category of D-branes is again a quotient

$$
\frac{\operatorname{DGr} S(\mathcal{W})}{T_{\Sigma}}
$$

where $T_{\Sigma}$ is a subcategory of $\operatorname{DGr} S(\mathcal{W})$ associated, via 20 , to modules annihilated by a power of the Cox ideal $B_{\Sigma}$.

\subsubsection{An example - the quintic threefold}

The previous discussion can be understood more easily in the context of an example. Let $V$ be the total space of the canonical line bundle over $\mathbb{P}^{4}$. We have a homogeneous coordinate ring

$$
S=\mathbb{C}\left[p, x_{0}, x_{1}, x_{2}, x_{3}, x_{4}\right],
$$

with respective grading $(-5,1,1,1,1,1)$. We have a superpotential

$$
\mathcal{W}=p\left(x_{0}^{5}+x_{1}^{5}+x_{2}^{5}+x_{3}^{5}+x_{4}^{5}\right) .
$$

The critical point set of $\mathcal{W}$ is the quintic hypersurface in $\mathbb{P}^{4}$ which we denote $X$.

In the Calabi-Yau phase the Cox ideal is $B_{\Sigma}=\left(x_{0}, \ldots, x_{4}\right)$. Let $\mathrm{w}$ be the $S$-module

$$
\mathbf{w}=\frac{S}{\left(x_{0}, \ldots, x_{4}\right)} .
$$

Since $\mathrm{w}$ is annihilated by $\mathcal{W}$ it may also be viewed as an $S^{\prime}$-module. We now compute a 
minimal free $S^{\prime}$-module resolution of w:

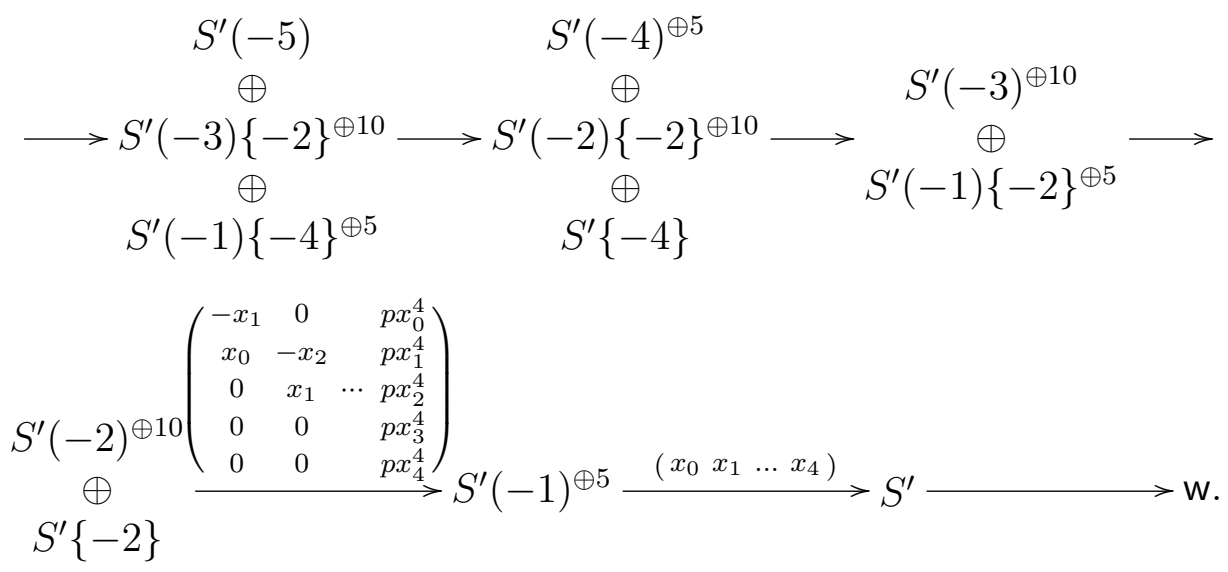

To write this infinite resolution as a matrix factorization we replace $S^{\prime}$ with $S$ and "roll it up" following $[12,14]$. w then corresponds to a $16 \times 16$ matrix factorization:

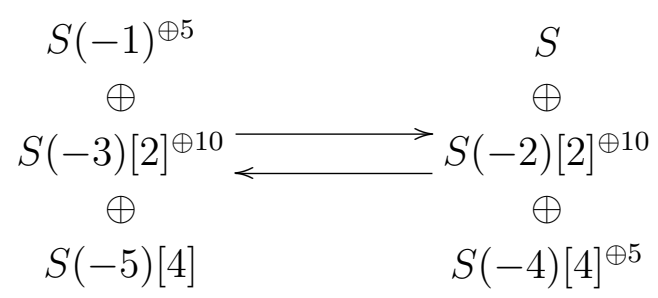

$T_{\Sigma}$ in 21) is generated by this matrix factorizations and all its shifts in both toric and homological grading. The category of D-branes on $X$ is given by the category of matrix factorizations $\operatorname{DGr} S(\mathcal{W})$ quotiented out by $T_{\Sigma}$.

From $[12,14,15]$ the category $\operatorname{DGr} S(\mathcal{W})$ is equivalent to the category of graded $A$-modules, where

$$
A=\frac{\mathbb{C}\left[x_{0}, x_{1}, \ldots, x_{4}\right]}{\left(x_{0}^{5}+x_{1}^{5}+\ldots+x_{4}^{5}\right)} .
$$

Serre's construction of sheaves on a projective variety then shows that the quotient (21) corresponds to the derived category of sheaves on the quintic threefold $X[11]$.

As an example of this equivalence let us consider the structure sheaf $\mathscr{O}_{X}$. This corresponds to the $A$-module $A$ itself. Let us denote $f=x_{0}^{5}+x_{1}^{5}+\ldots+x_{4}^{5}$. Following the algorithm in [14] we obtain the cyclic free $S^{\prime}$-resolution

$$
\longrightarrow S^{\prime}\{-4\} \stackrel{p}{\longrightarrow} S^{\prime}(-5)\{-2\} \stackrel{f}{\longrightarrow} S^{\prime}\{-2\} \stackrel{p}{\longrightarrow} S^{\prime}(-5) \stackrel{f}{\longrightarrow} S^{\prime} \longrightarrow A
$$

and so the structure sheaf $\mathscr{O}_{X}$ corresponds to the simplest matrix factorization

$$
S(-5) \frac{f}{<} S
$$

Let us use the notation $\mathrm{O}_{X}$ to refer to this particular matrix factorization form of $\mathscr{O}_{X}$. We may, of course, add (using mapping cones) anything in $T_{\Sigma}$ to this matrix factorization and still have a valid representative for $\mathscr{O}_{X}$. This fact will be used in section 2.2.4. 


\subsubsection{The monodromy ring}

Let us consider the K-theory and associated monodromy ring in the compact case. An object in $\mathbf{D}(\mathrm{gr}-S)^{\prime}$ typically has an infinite resolution in terms of free $S^{\prime}$-modules. Following section 2.1.1 we can associate to any object a power series which expresses the associated element of K-theory. As we shall see, it is very useful to include an extra variable to express the $R$-grading.

Let $P$ denote the ring of formal power series

$$
\mathbb{Z}\left[\left[s_{1}, s_{1}^{-1}, s_{2}, s_{2}^{-1}, \ldots, s_{r}, s_{r}^{-1}, \sigma, \sigma^{-1}\right]\right],
$$

and define a map on free $S^{\prime}$-modules

$$
k\left(S^{\prime}(\mathbf{v})\{m\}\right)=s_{1}^{v_{1}} s_{2}^{v_{3}} \ldots s_{r}^{v_{r}} \sigma^{-m} .
$$

By writing free $S^{\prime}$-module resolutions this extends to a map

$$
k: \mathbf{D}\left(\mathrm{gr}-S^{\prime}\right) \rightarrow P .
$$

Ultimately the resolution of any object in $\mathbf{D}\left(\mathrm{gr}-S^{\prime}\right)$ is periodic with period 2 and the product of two consecutive maps, lifted to an $S$-module map, is of homological degree (and hence $R$-degree since the two are identified) 2. It follows, that for any object a in $\mathbf{D}\left(\operatorname{gr}-S^{\prime}\right)$ we have

$$
\begin{aligned}
k(\mathrm{a}) & =f\left(s_{1}, s_{2}, \ldots, s_{r}, \sigma\right)\left(1+\sigma^{2}+\sigma^{4}+\ldots\right)+g\left(s_{1}, s_{2}, \ldots, s_{r}, \sigma\right) \\
& =\frac{f\left(s_{1}, s_{2}, \ldots, s_{r}, \sigma\right)}{1-\sigma^{2}}+g\left(s_{1}, s_{2}, \ldots, s_{r}, \sigma\right),
\end{aligned}
$$

where $f$ and $g$ are finite polynomials. From (20) we see that $f$ expresses the K-theory class of an object in $\operatorname{DGr} S(\mathcal{W})$ (with $\sigma$ set equal to one since the R-grading is equated to the homological grading).

In other words we have

Theorem 1 The map

$$
\kappa: \operatorname{Obj}(\operatorname{DGr} S(\mathcal{W})) \rightarrow \mathbb{Z}\left[s_{1}, s_{1}^{-1}, s_{2}, s_{2}^{-1}, \ldots, s_{r}, s_{r}^{-1}\right],
$$

given by

$$
\kappa(\mathrm{a})=\frac{1}{\pi i} \oint_{\gamma} k(\mathrm{a}) d \sigma
$$

where $\gamma$ is a small loop in the complex plane around $\sigma=1$, is a group homomorphism

$$
\kappa: K(\operatorname{DGr} S(\mathcal{W})) \rightarrow \mathbb{Z}\left[s_{1}, s_{1}^{-1}, s_{2}, s_{2}^{-1}, \ldots, s_{r}, s_{r}^{-1}\right] .
$$

This latter map will be injective but not surjective for all cases considered in this paper.

From (21) we see that there is a map from the K-theory associated to D-branes and a quotient of the polynomial ring $\mathbb{Z}\left[s_{1}, s_{1}^{-1}, s_{2}, s_{2}^{-1}, \ldots, s_{r}, s_{r}^{-1}\right]$.

One may also view K-theory directly in terms of the matrix factorization (16) as the class of $\bar{P}$ being given as the class of $P_{0}$ minus the class of $P_{1}$. However, since it is generally easier to compute this in terms of $S^{\prime}$-modules than matrix factorizations, the above theorem is more practical. 


\subsubsection{The quintic again}

Referring back to section 2.2 .2 we obtain

$$
\kappa(\mathbf{w})=\left(1-s^{-1}\right)^{5} .
$$

Since $w$ generates $T_{\Sigma}$ in the large radius Calabi-Yau phase, we define our monodromy ring for the quintic Calabi-Yau phase to be

$$
R_{0}(X)=\frac{\mathbb{Z}\left[s, s^{-1}\right]}{\left(\left(1-s^{-1}\right)^{5}\right)} .
$$

The subscript zero denotes the large radius phase. This is not, as a group, isomorphic to the $\mathrm{K}$-theory of $X$. The rank of $R_{0}$ is 5 whereas the rank of the $\mathrm{K}$-theory of $X$ is 4 .

For most purposes in this paper it will suffice to consider D-brane charge defined over the rationals. Algebraic K-theory coincides with topological K-theory for the quintic (since $\left.h^{1,0}=h^{2,0}=0\right)$ and topological K-theory over $\mathbb{Q}$ coincides with $H^{\text {even }}(X, \mathbb{Q})$ via the Chern character isomorphism. So, given the embedding

$$
i: X \rightarrow \mathbb{P}^{4},
$$

we have an isomorphism between the rational K-theory of $X$ and the pullback, via $i^{*}$ of the rational K-theory of $\mathbb{P}^{4}$. The latter is generated (redundantly) by $i^{*} \mathscr{O}_{\mathbb{P}^{4}}(m)=\mathscr{O}_{X}(m)$ for all $m \in \mathbb{Z}$.

Since $\kappa\left(\mathscr{O}_{X}\right)=1-s^{-5}$, the K-theory class of the D-brane $\mathscr{O}_{X}$ is represented in $R_{0}(X)$ by the equivalence class of $1-s^{5}$. Similarly $\mathscr{O}_{X}(m)$ corresponds to $\left(1-s^{-5}\right) s^{m}$.

Let $M_{0}^{\mathbb{Q}}(X)$ denote the rational K-theory of the quintic. Since monodromy acts on this we may regard $M_{0}^{\mathbb{Q}}(X)$ as a $R_{0}^{\mathbb{Q}}(X)$-module, where

$$
\begin{aligned}
R_{0}^{\mathbb{Q}}(X) & =R_{0}(X) \otimes_{\mathbb{Z}} \mathbb{Q} \\
& =\frac{\mathbb{Q}\left[s, s^{-1}\right]}{\left(\left(1-s^{-1}\right)^{5}\right)} .
\end{aligned}
$$

We therefore have the following

Theorem 2 The rational $K$-theory of the quintic, $M_{0}^{\mathbb{Q}}(X)$, as an $\mathbb{R}_{0}^{\mathbb{Q}}(X)$-module is given by the image of the map

$$
R_{0}^{\mathbb{Q}}(X) \stackrel{1-s^{-5}}{\longrightarrow} R_{0}^{\mathbb{Q}}(X) .
$$

Let $f(s)$ be in the kernel of the map in 410. Thus

$$
\begin{aligned}
f(s)\left(s^{5}-1\right) & =(s-1)^{5} g(s) \\
\text { i.e., } \quad f(s)\left(s^{4}+s^{3}+s^{2}+s+1\right) & =(s-1)^{4} g(s),
\end{aligned}
$$


for some Laurent polynomial $g(s)$. Multiplying out any negative powers of $s$ and using the fact that $\mathbb{Q}[s]$ is a unique factorization domain, $f(s)$ must have a factor of $(s-1)^{4}$. It follows that $M_{0}^{\mathbb{Q}}(X)$ is isomorphic to the cokernel of the man ${ }^{2}$

$$
R_{0}^{\mathbb{Q}}(X) \stackrel{\left(1-s^{-1}\right)^{4}}{\longrightarrow} R_{0}^{\mathbb{Q}}(X) .
$$

Note that as a vector space over $\mathbb{Q}, M_{0}^{\mathbb{Q}}(X)$ has dimension 4 as expected. The fact that $M_{0}^{\mathbb{Q}}(X)$ is annihilated by $(s-1)^{4}$ shows that monodromy around the large radius limit is unipotent of degree 4 as expected from [16].

The K-theory of $X$ is not given by the cokernel of $\left(1-s^{-1}\right)^{4}$ over the integers. In particular, the class of $\mathscr{O}_{X}$ is 5-divisible (see (50) ) in the cokernel of $\left(1-s^{-1}\right)^{4}$ but not in $K(X)$.

Now consider the Landau-Ginzburg phase. Here the Cox ideal is $B_{\Sigma}=(p)$. The cokernel of the map

$$
p: S(5)\{-2\} \rightarrow S
$$

is quasi-isomorphic to the complex

$$
\longrightarrow S\{-4\} \stackrel{p}{\longrightarrow} S\{-2\} \stackrel{f}{\longrightarrow} S(5)\{-2\} \stackrel{p}{\longrightarrow} S
$$

and so is associated to the matrix factorization (29) (shifted by [1]). This maps under $\kappa$ to $1-s^{5}$. Our monodromy ring is then

$$
R_{1}^{\mathbb{Q}}(X)=\frac{\mathbb{Q}\left[s, s^{-1}\right]}{\left(1-s^{-5}\right)}
$$

We can "transport" the D-brane $\mathscr{O}_{X}$ from the large radius Calabi-Yau phase to the Landau-Ginzburg phase using the trick from $[3,7]$. We use a tilting collection for the ambient toric variety $V$ of

$$
T=S \oplus S(-1) \oplus \ldots \oplus S(-4) .
$$

If we write a matrix factorization for a D-brane in the Calabi-Yau phase using only these summands (which may always be done) then exactly the same matrix factorization gives the same object in the Landau-Ginzburg phase.

As written, the matrix factorization $\mathrm{O}_{X}$ from (29) representing the 6-brane $\mathscr{O}_{X}$, is not in canonical form for transportation as it contains an $S(-5)$. To remedy this we use a mapping cone from the trivial object $\mathrm{w}$. To be precise, define $\mathscr{X}$ from

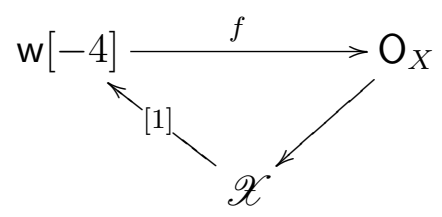

\footnotetext{
${ }^{2}$ We choose negative powers of $s$ to be consistent with the choice of tilting collections such as $(47)$.
} 
where $f$ contains the identity map $S(-5) \rightarrow S(-5)$ to cancel these terms away. Thus $\mathscr{X}$ is the "correct" representative for $\mathscr{O}_{X}$.

Now, in the Landau-Ginzburg phase, it is $\mathrm{O}_{X}$ which is trivial while $\mathrm{w}$ is not. Writing (48) as

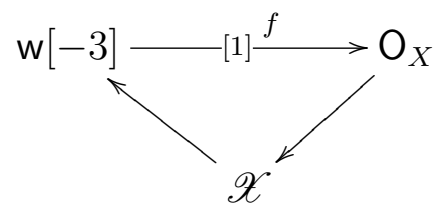

we obtain an equivalence between $\mathscr{X}$ and $\mathrm{w}[-3]$ in the Landau-Ginzburg phase. In other words, $\mathscr{O}_{X}$ is represented by $\mathrm{w}[-3]$ in the Landau-Ginzburg phase as also argued in [17].

In the monodromy ring $R_{0}^{\mathbb{Q}}(X)$, this tilting collection can be expressed in terms of a Gröbner basis. To do this we introduce a new variable $t=s^{-1}$. In particular if we use lexicographical ordering on the ring $\mathbb{Q}[s, t]$ then a Gröbner basis of the ideal $\left(s t-1,(1-t)^{5}\right)$ is has leading monomials $t^{5}$ and $s$. Thus, any polynomial in the quotient ring (40) put in normal form using this basis will have monomials only in the set $\left\{1, t, \ldots, t^{4}\right\}$ exactly corresponding to summands of (47). The same is true for the Landau-Ginzburg phase and the corresponding ideal $\left(s t-1,1-t^{5}\right)$.

In particular, the normal form of $1-s^{-5}$, the charge of the 6-brane $\mathscr{O}_{X}$, is

$$
5 t-10 t^{2}+10 t^{3}-5 t^{4} \text {. }
$$

Correspondingly, in the Landau-Ginzburg phase, the charge of $w[-3]$ is given by $-(1-t)^{5}$. This has normal form with respect to the ideal $\left(s t-1,1-s^{5}\right)$ also given by (50) as we would expect.

In the Landau-Ginzburg phase the K-theory spanned by the D-brane $\mathscr{O}_{X}$ and its images under monodromy around the Gepner point are therefore given by the image of $\left(1-s^{-1}\right)^{5}$. Let $M_{1}^{\mathbb{Q}}(X)$ denote this $R_{1}^{\mathbb{Q}}(X)$-module. It follows that $M_{1}^{\mathbb{Q}}(X)$ is the cokernel of

$$
R_{1}^{\mathbb{Q}}(X) \stackrel{1+s^{-1}+s^{-2}+s^{-3}+s^{-4}}{\longrightarrow} R_{1}^{\mathbb{Q}}(X) .
$$

Since this is rank 4 it spans the full K-theory of D-branes in the Landau-Ginzburg phase. It also shows that the monodromy about the Gepner point is $\mathbb{Z}_{5}$ and that no periods (linear maps $K(X) \rightarrow \mathbb{C}$ ) are invariant under this monodromy, as can be shown by direct computation of the periods [6].

\section{True Hybrid Model Phases}

\subsection{The hybrid phase}

A simple model with a hybrid phase is a gauged linear $\sigma$-model with one gauge symmetry, under which the fields $p_{0}, p_{1}, x_{0}, x_{1}, \ldots, x_{5}$ have charges $(-3,-3,1,1, \ldots, 1)$ respectively. The worldsheet superpotential is

$$
\mathcal{W}=p_{0} f_{0}\left(x_{i}\right)+p_{1} f_{1}\left(x_{i}\right)
$$


where $f_{0}\left(x_{i}\right)$ and $f_{1}\left(x_{i}\right)$ are homogeneous polynomials of degree 3 in $x_{0}, \ldots, x_{5}$. We do not need to know any details about $f_{0}\left(x_{i}\right)$ and $f_{1}\left(x_{i}\right)$ except that they are sufficiently generic so that $X$ is smooth. As an example, one could use [18]

$$
\begin{aligned}
& f_{0}=x_{0}^{3}+x_{1}^{3}+x_{2}^{3}+x_{3} x_{4} x_{5} \\
& f_{1}=x_{3}^{3}+x_{4}^{3}+x_{5}^{3}+x_{0} x_{1} x_{2} .
\end{aligned}
$$

For $r \gg 1 B_{\Sigma}=\left(x_{0}, x_{1}, \ldots, x_{5}\right)$ and the low-energy theory is a nonlinear $\sigma$-model on the intersection $f_{0}=f_{1}=0$ in $\mathbb{P}^{5}$. This is a typical Calabi-Yau phase. The non-anomalous $R$ symmetry under which $p_{a}$ have charge 2 and $x_{i}$ are invariant is unbroken by the expectation values of $x_{i}$ and survives as the superconformal $U(1)_{R}$ in the low-energy limit.

For $r \ll-1 B_{\Sigma}=\left(p_{0}, p_{1}\right)$. The gauge symmetry is broken to a $\mathbb{Z}_{3}$ subgroup by the expectation values of $p_{a}$. Classical vacua are constrained to lie at $x_{i}=0$ by the $F$-terms, so the space of classical vacua is $\mathbb{P}^{1}$ and classically the radius of this is $-r$, so that naïvely in the limit we have a large-radius, weakly coupled nonlinear sigma model on this target space. These vacua are invariant under the $R$-symmetry to within a gauge transformation. To see this explicitly, combine an $R$-rotation with a gauge transformation such that the $p_{a}$ are left invariant. Under this transformation the $x_{i}$ all have charge $2 / 3$. A nonlinear $\sigma$-model on $\mathbb{P}^{1}$ cannot be the complete story, of course, and indeed the fields $x_{i}$, while obstructed from acquiring nonzero expectation values, are massless and cannot be integrated away. Considering fluctuations about a generic classical vacuum, we find that in addition to the massless fluctuation tangent to $\mathbb{P}^{1}$ there are six massless superfields $x_{i}$ interacting via a cubic superpotential and transforming under the unbroken discrete gauge symmetry, describing a Landau-Ginzburg orbifold theory with central charge $\hat{c}=2$. We can imagine the hybrid theory as a fibration of a Landau-Ginzburg orbifold over a $\mathbb{P}^{1}$ base, in analogy with the construction of Calabi-Yau spaces as fibrations over $\mathbb{P}^{1}$ with a K3 fibre.

Let us consider the Landau-Ginzburg theory we find in a particular fibre, i.e., fix the value of $p_{0}$ and $p_{1}$. We then have some cubic equation in 6 variables. This is a $\hat{c}=2$ superconformal theory with integral $U(1)_{R}$ charges (the $\mathbb{Z}_{3}$ orbifold projects out all states with fractional charge) and so must be equivalent [19] to a compactification on either a four-torus or a K3 surface. In the case at hand, it is easy to compute that this has 20 deformations of complex structure from deforming the cubic equation and so must be a rather curious realization of a $\mathrm{K} 3$ as noted in [20].

Naïvely one might try to identify this "K3 surface" with a cubic hypersurface in $\mathbb{P}^{5}$. This correspondence is exactly the analogue of identifying the mirror of the Z-orbifold as a cubic equation in $\mathbb{P}^{8}$ as was done in $[21,22]$. Probably a more useful interpretation in the current context is the following. Since we have defined our conformal field theory in terms of a superpotential it must be "algebraic" in some sense. Since 20 deformations of complex structure are manifest in the deformation of this defining equation, it must have Picard number $\rho=20-20=0$ (see [23], for example). So the fibre should be thought of as an algebraic K3 surface with Picard number zero.

Even though this description is something of an oxymoron it serves our model quite well. If one uses such $\rho=0 \mathrm{~K} 3$ surfaces as fibres in a K3-fibration of a Calabi-Yau threefold then 
there will be no deformations of $B+i J$ associated with Kähler form deformations of the fibre. In particular there is no deformation that can blow the fibre up into a geometrical large radius K3 surface. Our model has no true geometric K3-fibration phase. Instead, the "K3-fibre" is stuck at the $\alpha^{\prime}$ scale in the exact same way as the mirror of the Z-orbifold is stuck at some small scale.

This hybrid model was also discussed in [5], where the fibres were interpreted as "noncommutative" K3s.

It is perhaps worth noting here that the cubic superpotential degenerates at nine points on $\mathbb{P}^{1}$. These do not correspond to singular conformal theories in the same way that singular fibres do not imply a singularity in the total space of a fibration. Here this is very explicitly clear from the Lagrangian: when the cubic superpotential is degenerate the pure LandauGinzburg theory acquires a flat direction. In the hybrid model, this is lifted by the $F$-terms given by derivatives of $\mathcal{W}$ with respect to $p_{a}$ so there is no decompactification of the space of vacua and no singularity.

\subsection{The monodromy ring}

Let us apply the methods of section 2 to this model. The homogeneous coordinate ring of the ambient toric variety is

$$
S=\mathbb{C}\left[p_{0}, p_{1}, x_{0}, x_{1}, x_{2}, x_{3}, x_{4}, x_{5}\right]
$$

with respective grading $(-3,-3,1,1, \ldots, 1)$. We use the Calabi-Yau $R$-charge assignments, so that both $p_{0}$ and $p_{1}$ have charge 2 while the $x_{i}$ 's all have charge 0 .

As described above, this model has two phases and is similar to the quintic as described in section 2.2 in many ways. In the Calabi-Yau phase the Cox ideal is $\left(x_{0}, \ldots, x_{5}\right)$. Again we define the $S$-module

$$
\mathrm{w}=\frac{S}{\left(x_{0}, \ldots, x_{5}\right)},
$$

which is a no-brane in this phase. In analogy with section 2.2.2 (and as computed in [24]) we associate the following matrix factorization to w:

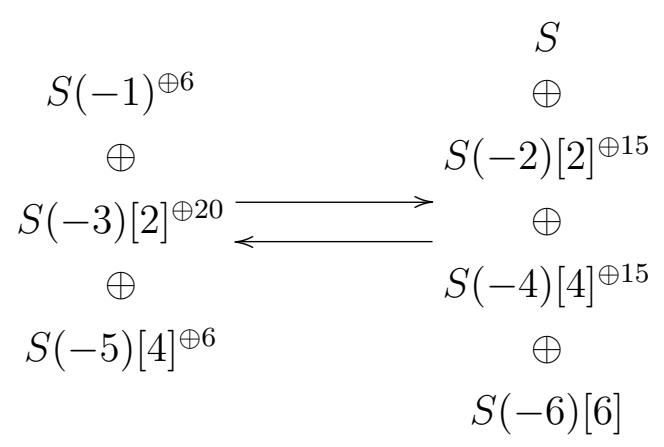

We now have

$$
\kappa(\mathbf{w})=\left(1-s^{-1}\right)^{6}
$$


and so the monodromy ring in the Calabi-Yau phase is

$$
R_{0}^{\mathbb{Q}}=\frac{\mathbb{Q}\left[s, s^{-1}\right]}{\left(\left(1-s^{-1}\right)^{6}\right)} .
$$

Thus the sheaf $\mathscr{O}_{X}$ is associated with the $S$-module $O_{X}$ defined as the cokernel of the map.

$$
S(-3)^{\oplus 2} \stackrel{f_{0}, f_{1}}{\longrightarrow} S
$$

$\mathrm{O}_{X}$ is an $S^{\prime}$-module since it is annihilated by $W$. This can be repackaged as the matrix factorization [24]:

$$
\begin{aligned}
& \begin{array}{c}
S(-3) \quad\left(\begin{array}{cc}
f_{0} & f_{1} \\
-p_{1} & p_{0}
\end{array}\right) \quad S(0) \\
\oplus \longleftarrow
\end{array} \\
& S(-3) \quad\left(\begin{array}{cc}
p_{0} & -f_{1} \\
p_{1} & f_{0}
\end{array}\right) \quad S(-6)[2]
\end{aligned}
$$

and

$$
\kappa\left(\mathrm{O}_{X}\right)=\left(1-s^{-3}\right)^{2} .
$$

As for the quintic we define the module $M_{0}^{\mathbb{Q}}(X)$ as the image of $\left(1-s^{-3}\right)^{2}$. This is isomorphic to the cokernel of the map $\left(1-s^{-1}\right)^{4}$. Thus we recover maximal unipotency at the large radius limit as expected.

For the hybrid phase $B_{\Sigma}=\left(p_{0}, p_{1}\right)$ and it is $\mathrm{O}_{X}$ that becomes the generating no-brane. We then have a monodromy ring

$$
R_{1}^{\mathbb{Q}}=\frac{\mathbb{Q}\left[s, s^{-1}\right]}{\left(\left(1-s^{-3}\right)^{2}\right)} .
$$

Using a tilting object

$$
T=S \oplus S(-1) \oplus \ldots \oplus S(-5)
$$

we may transport the D-brane $\mathscr{O}_{X}$ from the Calabi-Yau phase to the hybrid phase in a manner identical to the construction for the quintic in section 2.2.4. This gives an equivalence

$$
\mathscr{O}_{X} \sim \mathrm{w}[-3]
$$

Thus we know that the K-theory of the hybrid phase contains an $R_{1}^{\mathbb{Q}}$ given by the image of $\kappa(\mathrm{w})=\left(1-s^{-1}\right)^{6}$. The image of $\left(1-s^{-1}\right)^{6}$ is isomorphic to the cokernel of

$$
\left(1+s+s^{2}\right)^{2}
$$

This is rank 4 and so is the full K-theory for the hybrid phase and we denote it $M_{1}^{\mathbb{Q}}(X)$.

The form of 65 immediately yields the monodromy of periods around the hybrid limit point.

We view $M_{1}^{\mathbb{Q}}(X)$ as the quotient of $R_{1}^{\mathbb{Q}}(X)$ by $\left(1+s+s^{2}\right)^{2}$ and define the submodule $N \subset M_{1}^{\mathbb{Q}}$ as elements of the form $\left(1+s+s^{2}\right) f(s)$. This is a rank two submodule. Writing

$$
s^{3}=1-(1-s)\left(1+s+s^{2}\right)
$$

we see that monodromy on periods three times around the limit point for an element in $N$ is the identity while the other periods add on something in $N$. We discuss these periods more in section 3.4 . 


\subsection{The symplectic inner product}

The lattice of D-brane charges has a symplectic inner product. For A-branes on a CalabiYau threefold this is nothing more than the intersection inner product between 3-cycles. For B-branes corresponding to objects $\mathscr{E} \bullet$ and $\mathscr{F} \bullet$ in $\mathbf{D}(X)$ this corresponds to

$$
\begin{aligned}
\left\langle\mathscr{E}^{\bullet}, \mathscr{F}^{\bullet}\right\rangle & =\sum_{i}(-1)^{i} \operatorname{dim} \operatorname{Ext}^{i}\left(\mathscr{E} \bullet, \mathscr{F}^{\bullet}\right) \\
& =\int_{X} \operatorname{ch}\left(\mathscr{E}^{\bullet}\right)^{\vee} \wedge \operatorname{ch}\left(\mathscr{F}^{\bullet}\right) \wedge t d\left(T_{X}\right) .
\end{aligned}
$$

Consider first the linear map from K-theory to $\mathbb{Z}$ given by Hirzebruch-Riemann-Roch

$$
\begin{aligned}
\langle\mathscr{E} \bullet & =\sum_{i}(-1)^{i} \operatorname{dim} H^{i}\left(\mathscr{E}^{\bullet}\right) \\
& =\int_{X} \operatorname{ch}(\mathscr{E} \bullet) \wedge t d\left(T_{X}\right) .
\end{aligned}
$$

We may regard this as a linear map from $R(X)$ to $\mathbb{Z}$. This map can be explicitly determined in the large radius phase since we know $\mathscr{O}_{X}(n)$ corresponds to $s^{n}\left(1-s^{-3}\right)^{2}$ in $R(X)$; and we know the corresponding value in $\mathbb{Z}$. We need only evaluate at $\operatorname{rank} K(X)=4$ points to determine the map fully. The result is surprisingly simple:

$$
\langle\mathscr{E} \bullet\rangle=\left.\mathrm{NF}(\kappa(\mathscr{E} \bullet))\right|_{s^{0}},
$$

where NF denotes the normal form of the polynomial with respect to the Gröbner basis associated to the tilting collection (63), i.e., the monomials are of the form $s^{0}, s^{-1}, \ldots, s^{-5}$. The subscript $s^{0}$ means take the coefficient of $s^{0}$. Now $c h$ is a ring isomorphism between $K(X)$ and cohomology, and $\mathscr{O}_{X}(n)^{\vee}=\mathscr{O}_{X}(-n)$. It follows that

$$
\left\langle\mathscr{E}^{\bullet}, \mathscr{F}^{\bullet}\right\rangle=\left.\mathrm{NF}\left(\left(\frac{\kappa\left(\mathscr{E}^{\bullet}\right)}{\left(1-s^{-3}\right)^{2}}\right)^{\vee} \kappa\left(\mathscr{F}^{\bullet}\right)\right)\right|_{s^{0}}
$$

where $f(s)^{\vee}=f\left(s^{-1}\right)$.

The symplectic form is invariant over the moduli space and so we may compute it in any phase by transporting D-branes back into the large radius limit phase and using (70). For example, in the hybrid phase we may choose a basis $1, s^{-1}, s^{-2}, s^{-3}$ for the module $M_{1}^{\mathbb{Q}}(X)$. With respect to this basis the symplectic product is given by

$$
\left(\begin{array}{rrrr}
0 & -6 & 15 & -18 \\
6 & 0 & -6 & 15 \\
-15 & 6 & 0 & -6 \\
18 & -15 & 6 & 0
\end{array}\right)
$$




\subsection{Periods and special Kähler geometry}

Until now we have avoided using the period approach to analyzing monodromy, etc. Instead we can derive all the information we need to know about the periods from the algebraic methods above. This allows us to understand issues about the moduli space metric and asymptotic forms of the D-brane masses without having to solve any differential equations.

$M_{i}^{\mathbb{Q}}(X)$ are modules over the ring $R_{i}^{\mathbb{Q}}(X)$. In the case that the moduli space is onedimensional $R_{i}^{\mathbb{Q}}(X)$ is a principal ideal domain. This allows us to use the well-known classification of modules over P.I.D.'s and, in particular, the notation of rational canonical form (see, for example, chapter 12 of [25]).

Periods are an element of the dual module

$$
Z \in \operatorname{Hom}_{R_{i}^{\mathbb{Q}}}\left(M_{i}^{\mathbb{Q}}(X), \mathbb{C}\right) .
$$

If $M_{i}^{\mathbb{Q}}(X)$ is the cokernel of the polynomial $q \in R_{i}^{\mathbb{Q}}(X)$ then monodromy around the limit point expressed as a matrix is simply the companion matrix for $q$. The asymptotic form of the periods, can then by simply deduced from the Jordan canonical form of this matrix.

For example, consider the hybrid phase of the $X$, the bicubic in $\mathbb{P}^{5}$. We may put $q=$ $\left(1+s+s^{2}\right)^{2}$ and then the Jordan canonical form of the companion matrix is

$$
\left(\begin{array}{cccc}
\omega & 1 & & \\
0 & \omega & & \\
& & \omega^{2} & 1 \\
& & & \omega^{2}
\end{array}\right)
$$

Let $z$ be a coordinate on the moduli space such that the limit point is $z=0$. The asymptotic form of the periods as $z \rightarrow 0$ are then

$$
\begin{aligned}
& \Phi_{1}=z^{\frac{1}{3}}+O\left(z^{\frac{4}{3}}\right) \\
& \Phi_{2}=\frac{1}{2 \pi i} z^{\frac{1}{3}} \log (z)+O\left(z^{\frac{1}{3}}\right) \\
& \Phi_{3}=z^{\frac{2}{3}}+O\left(z^{\frac{5}{3}}\right) \\
& \Phi_{4}=\frac{1}{2 \pi i} z^{\frac{2}{3}} \log (z)+O\left(z^{\frac{2}{3}}\right)
\end{aligned}
$$

It is important to note that the Jordan canonical form cannot be acquired from the rational canonical form working over $\mathbb{Q}$ - one must use complex numbers numbers. This means that the periods of the pure form (74) are not realized by any actual D-brane. Instead we only have certain nontrivial linear combinations. In particular, looking back at the discussion in section 3.2 we have any periods associated to objects in $N \subset M_{1}^{\mathbb{Q}}(X)$ going as $z^{\frac{1}{3}}+O\left(z^{\frac{2}{3}}\right)$ and any other period going as $\frac{1}{2 \pi i} z^{\frac{1}{3}} \log (z)+O\left(z^{\frac{2}{3}} \log (z)\right)$.

Periods give the central charge of a D-brane up to some normalization. This normalization comes from special Kähler geometry. Let $\alpha_{i}$ and $\beta^{i}$ form a basis for $M_{1}^{\mathbb{Q}}(X)$ (as a vector space) such that $i$ runs from 1 to half the dimension and the symplectic form satisfies

$$
\left\langle\alpha_{i}, \beta^{j}\right\rangle=\delta_{i}^{j} .
$$


Then define the Kähler potential [26]

$$
K=-\log \left(2 \sum_{i} \operatorname{Im}\left(Z\left(\alpha_{i}\right) \bar{Z}\left(\beta^{i}\right)\right)\right) .
$$

In the hybrid limit in our example we have

$$
e^{-K} \sim|z|^{\frac{2}{3}} \log |z|, \quad \text { as } z \rightarrow 0 .
$$

The metric on the moduli space is then given by

$$
\begin{aligned}
g_{z \bar{z}} & =\partial \bar{\partial} K \\
& \sim \frac{1}{(|z| \log |z|)^{2}} \quad \text { as } z \rightarrow 0 .
\end{aligned}
$$

This implies that the limit point $z=0$ is an infinite distance away in the moduli space. This in turn implies that we should view this limit as some kind of decompactification.

The mass of a D-brane is given by

$$
m=\frac{e^{\frac{K}{2}}|Z|}{g_{10}},
$$

where $g_{10}$ is the 10-dimensional string coupling. If we go to a decompactification limit it is natural to hold the 4-dimensional string coupling, $g_{4}$, constant. In this case we get

$$
m=\frac{1}{g_{4}} e^{\frac{K}{2}}|Z| \sqrt{\operatorname{Vol}(X)} .
$$

In the usual decompactification limit of a large radius Calabi-Yau threefold one then finds using (80) that the 0-brane has a mass that is constant, while 2-branes have mass scaling with the Kähler form, 4-branes have mass scaling with the square of the Kähler form, etc.

In our hybrid limit we have

$$
e^{\frac{K}{2}}|Z(a)| \sim \frac{1}{\sqrt{\log |z|}},
$$

for elements $a \in N$, while

$$
e^{\frac{K}{2}}|Z(a)| \sim \sqrt{\log |z|},
$$

for elements $a \in M_{1}^{\mathbb{Q}}(X), a \notin N$.

There is an obvious interpretation of this behaviour in terms of a decompactification. We assert that $\operatorname{Vol}(X)$ scales as $\log |z|$. Then we see that elements of $N$ will have masses that are constant in the limit $z \rightarrow 0$ and so look like 0-branes. Other elements of $M_{1}^{\mathbb{Q}}(X)$ have masses scaling like $\log |z|$ and are 2-branes. Since the volume of $X$ itself scales like $\log |z|$, it seems that $X$ itself is 2-dimensional. The Kähler form on $X$ looks like a ratio of periods:

$$
B+i J=\frac{1}{2 \pi i} \log (z)+\ldots,
$$




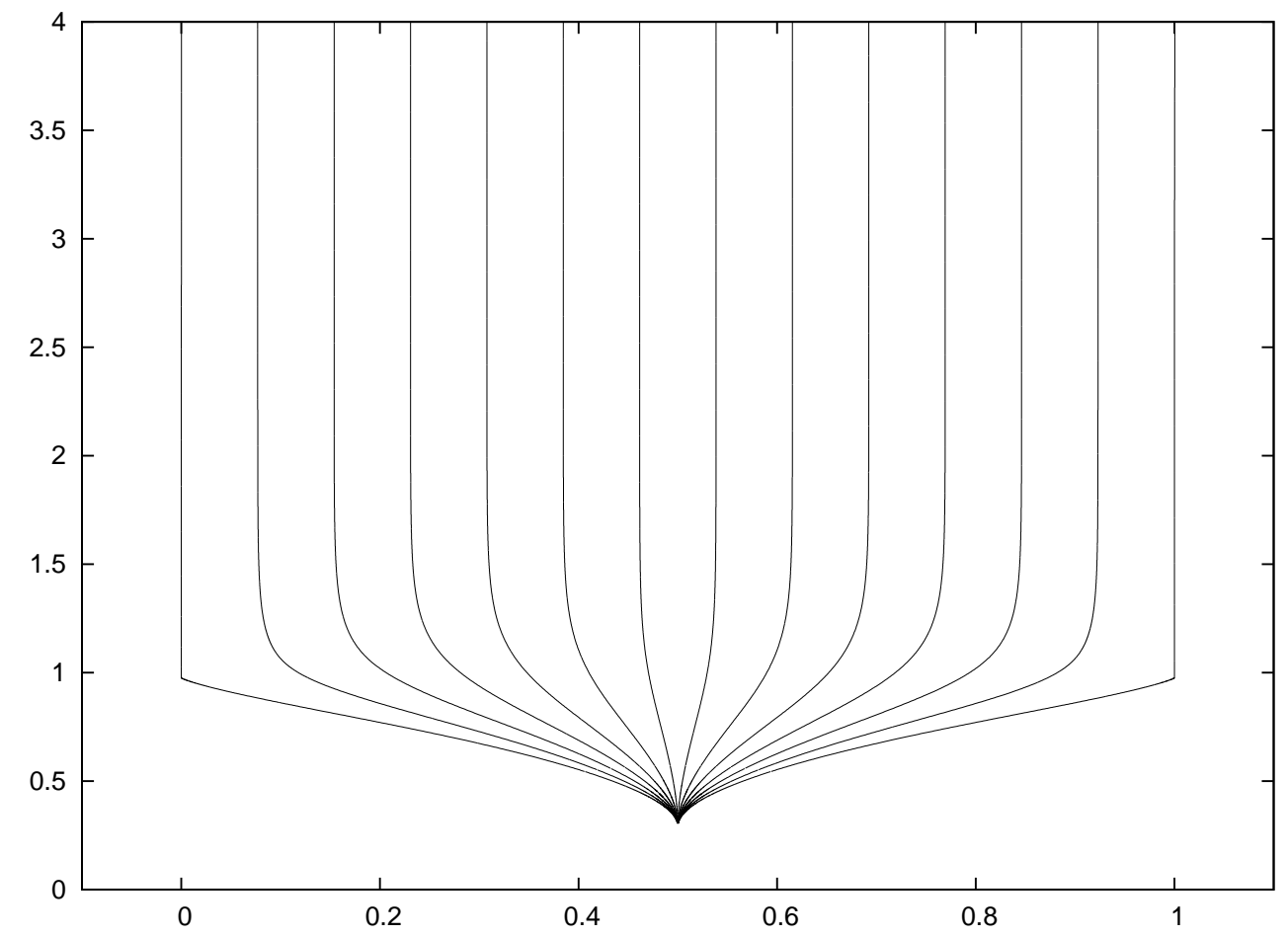

Figure 1: Hybrid Moduli Space for $B+i J$.

as usual.

This is entirely what would expect from the linear $\sigma$-model picture of the hybrid limit. The base $\mathbb{P}^{1}$ becomes infinitely large in the limit. The interpretation of the hybrid limit in terms of a fibration is in agreement with this. Because the "K3 fibres" lack holomorphic curves, we are naturally led to interpret the instanton expansion about the hybrid limit as an expansion in worldsheet instantons wrapping the base space.

On the other hand, analytic continuation of the worldsheet instanton series from the Calabi-Yau phase (where they correspond to Gromov-Witten invariants) as was done in $[27,28]$ might suggest a different interpretation. Here one analytically continues the ratio of periods representing the Kähler form in the large radius Calabi-Yau phase. The resulting representation of the moduli space is shown in figure 1 and is the analogue of the "scorpion" diagram of [6]. The cusp at $B+i J=\frac{1}{2}+\frac{i}{2 \sqrt{3}}$ represents the hybrid model limit and it would appear, at first sight, to represent a Calabi-Yau at finite size. Unfortunately, the true metric in the moduli space becomes very singular at the cusp and so figure 1 is misleading.

Let us emphasize that what we are calling "0-branes" in the hybrid limit are not at all the same D-branes that were 0-branes in the large Calabi-Yau phase limit. The Calabi-Yau 0-branes do not have D-brane charge in $N$ and so become infinitely massive in the hybrid limit. Conversely, the 0-branes of the hybrid model look 6-dimensional in the Calabi-Yau phase [24] and have divergent masses in the large radius limit. 


\subsection{0-Brane probes}

Having established the K-theory classes of the candidate 0-branes in the hybrid limit we would like to explicitly see the corresponding objects in the category of D-branes. The candidate 0-branes were proposed in [24] and these turn out to be entirely consistent with the above.

Define objects

$$
\mathscr{P}_{\left[x_{0}, x_{1}\right]}=\text { Cone }\left(\mathrm{w}(3)[-2] \stackrel{x_{0} p_{1}-x_{1} p_{0}}{\longrightarrow} \mathrm{w}\right),
$$

where $x_{0}$ and $x_{1}$ are complex numbers. If $\left[x_{0}, x_{1}\right]=[0,0]$ then this object is unstable [24]. It is also easy to prove an isomorphism

$$
\mathscr{P}_{\left[x_{0}, x_{1}\right]} \cong \mathscr{P}_{\left[\lambda x_{0}, \lambda x_{1}\right]}^{0},
$$

where $\lambda \in \mathbb{C}^{*}$. Thus the objects $\mathscr{P}_{\left[x_{0}, x_{1}\right]}$ are naturally parametrized by points $\left[p_{0}, p_{1}\right]=$ $\left[x_{0}, x_{1}\right]$ on the $\mathbb{P}^{1}$ of the hybrid model.

Clearly

$$
\kappa\left(\mathscr{P}_{\left[x_{0}, x_{1}\right]}\right)=\left(1-s^{3}\right) \kappa(\mathrm{w}),
$$

and so $\kappa\left(\mathscr{P}_{\left[x_{0}, x_{1}\right]}\right)$ is a multiple of $\left(1-s^{3}\right)$ in $M_{1}^{\mathbb{Q}}(X)$. Thus $\kappa\left(\mathscr{P}_{\left[x_{0}, x_{1}\right]}\right)$ lies in $N$ and has the right D-brane charge to be a 0 -brane from the previous section.

In [24] it was shown that $\mathscr{P}_{\left[x_{0}, x_{1}\right]}$ is stable in the hybrid limit, at least with respect to the obvious decay mode.

It was also shown in [24] that, in the large radius phase language, the object $\mathscr{P}_{\left[x_{0}, x_{1}\right]}$ corresponds to the complex of sheaves

$$
\text { Cone }\left(\left.\Omega_{\mathbb{P}^{5}}^{2}\right|_{X}(3)[1] \rightarrow \mathscr{O}_{X}[3]\right) .
$$

Computing the Chern character of $\mathscr{P}_{\left[x_{0}, x_{1}\right]}$ in terms of the cohomology of $X$ yields

$$
\operatorname{ch}\left(\mathscr{P}_{\left[x_{0}, x_{1}\right]}\right)=9+6 x-x^{3},
$$

where $x$ is the generator of $H^{2}\left(\mathbb{P}^{5} ; \mathbb{Z}\right)$ restricted to $X$. According to the Lefschetz hyperplane theorem, 1 and $x$ generate $H^{0}(X ; \mathbb{Z})$ and $H^{2}(X ; \mathbb{Z})$ respectively. But $x^{3}$ is Poincaré dual to 9 points on $X$ and thus $x^{3} / 9$ generates $H^{6}(X ; \mathbb{Z})$. It follows that $\operatorname{ch}\left(\mathscr{P}_{\left[x_{0}, x_{1}\right]}\right)$ is actually 3-divisible in the lattice of D-brane charges.

This raises the possibility that $\mathscr{P}_{\left[x_{0}, x_{1}\right]}$ is actually a polystable object which is a direct sum of three objects with the same D-brane charge. This would imply that $\mathscr{P}_{\left[x_{0}, x_{1}\right]}$ is actually three 0 -branes rather than one. We can show this is not the case by computing $\operatorname{Hom}\left(\mathscr{P}_{\left[x_{0}, x_{1}\right]}, \mathscr{P}_{\left[x_{0}, x_{1}\right]}\right)$ from (87). Using Macaulay 2 for the form (53) one may compute

$$
\begin{aligned}
& \operatorname{dim} \operatorname{Ext}^{1}\left(\left.\Omega_{\mathbb{P}^{5}}^{2}\right|_{X}(3), \mathscr{O}_{X}\right)=0 \\
& \operatorname{dim} \operatorname{Ext}^{2}\left(\left.\Omega_{\mathbb{P}^{5}}^{2}\right|_{X}(3), \mathscr{O}_{X}\right)=2 .
\end{aligned}
$$

A standard application of long exact sequences then yields

$$
\operatorname{dim} \operatorname{Hom}\left(\mathscr{P}_{\left[x_{0}, x_{1}\right]}, \mathscr{P}_{\left[x_{0}, x_{1}\right]}\right)=1,
$$


showing that $\mathscr{P}_{\left[x_{0}, x_{1}\right]}$ is not a direct sum of objects.

Since $\mathscr{P}_{\left[x_{0}, x_{1}\right]}$ are light in the hybrid limit, apparently stable and irreducible we therefore assert that they are indeed the 0-branes that probe the decompactification geometry of the hybrid limit. From now on we will assume this assertion is true.

Now consider

$$
\mathscr{P}_{\left[x_{0}, x_{1}\right]}(n)=\text { Cone }\left(\mathrm{w}(n+3)[-2] \stackrel{x_{0} p_{1}-x_{1} p_{0}}{\longrightarrow} \mathrm{w}(n)\right) .
$$

Monodromy around the hybrid limit point will transform

$$
\mathscr{P}_{\left[x_{0}, x_{1}\right]}(n) \rightarrow \mathscr{P}_{\left[x_{0}, x_{1}\right]}(n+1) .
$$

The D-brane charges of $\mathscr{P}_{\left[x_{0}, x_{1}\right]}(n)$ and $\mathscr{P}_{\left[x_{0}, x_{1}\right]}(n+1)$ differ. This means, unlike a conventional large radius limit, the 0-branes transform into other D-branes under the " $B$-field" shift $B \rightarrow B+1$. The objects $\mathscr{P}_{\left[x_{0}, x_{1}\right]}(n)$ would appear to have equal right to be called a 0 -brane for any value of $n$.

Since the Cox ideal in the hybrid phase is $\left(p_{0}, p_{1}\right)$, we have an exact sequence

$$
\mathrm{w}(n+6)[-4] \stackrel{\left(\begin{array}{c}
-p_{1} \\
p_{0}
\end{array}\right)}{\longrightarrow} \mathrm{w}(n+3)[-2]^{\oplus 2} \stackrel{\left(p_{0} p_{1}\right)}{\longrightarrow} \mathrm{w}(n) .
$$

This implies

$$
\text { Cone }\left(\mathscr{P}_{\left[x_{0}, x_{1}\right]}(n+3)[-2] \stackrel{f}{\longrightarrow} \mathscr{P}_{\left[x_{0}, x_{1}\right]}(n)\right)=0,
$$

where $f$ corresponds to any linear combination of $p_{0}$ and $p_{1}$ other than $x_{0} p_{1}-x_{1} p_{0}$. In other words, we have

Theorem 3 There is an isomorphism in the category of D-branes:

$$
\mathscr{P}_{\left[x_{0}, x_{1}\right]}(n+3) \cong \mathscr{P}_{\left[x_{0}, x_{1}\right]}(n)[2] .
$$

Now $\mathscr{P}_{\left[x_{0}, x_{1}\right]}[2]$ and $\mathscr{P}_{\left[x_{0}, x_{1}\right]}$ correspond to the same physical D-brane [10,29]. So going three times around the hybrid limit produces trivial physical monodromy on the 0-branes. Note that we already knew that this was true for D-brane charge since we observed in section 3.4 that objects in $N$ were associated to periods of the form $z^{\frac{1}{3}}$. However, theorem 3 is much stronger since it says that a 0 -brane at a given point on the $\mathbb{P}^{1}$ comes back to the same 0-brane at the same point.

Theorem 3 shows that there are three 0 -brane-like objects for each point on $\mathbb{P}^{1}$. Each of these 0-branes has a different charge. 


\subsection{Four quadrics on $\mathbb{P}^{7}$.}

Consider another model with a one-dimensional moduli space and a hybrid phase which is very similar to the example above. Let $X$ be the intersection of four generic quadric polynomials in $\mathbb{P}^{7}$. This was studied in [4] and more recently in [5].

The homogeneous coordinate ring is

$$
S=\mathbb{C}\left[p_{0}, p_{1}, p_{2}, p_{3}, x_{0}, \ldots, x_{7}\right],
$$

with respective grading $(-2,-2,-2,-2,1,1, \ldots, 1)$. The $R$-charge of each $p_{j}$ is set to 2 while the $x_{i}$ 's are all R-charge 0 . The worldsheet superpotential is

$$
\mathcal{W}=p_{0} f_{0}\left(x_{i}\right)+\ldots+p_{3} f_{3}\left(x_{i}\right),
$$

where $f_{j}\left(x_{i}\right)$ are homogeneous polynomials of degree 2 in $x_{0}, \ldots, x_{7}$ and are sufficiently generic so that $X$ is smooth.

The large radius Calabi-Yau phase corresponds to $X$. In the hybrid phase $B_{\Sigma}=$ $\left(p_{0}, \ldots, p_{3}\right)$. The geometrical interpretation of this phase in terms of the gauged linear $\sigma$-model is that of a $\mathbb{P}^{3}$, with homogeneous coordinates $\left[p_{0}, \ldots, p_{3}\right]$. Over each point in this $\mathbb{P}^{3}$ we have a Landau-Ginzburg theory with fields $x_{0}, \ldots, x_{7}$ and a quadric superpotential. At first sight, a Landau-Ginzburg theory with quadric superpotential is completely trivial and so the target space should be simply $\mathbb{P}^{3}$. This cannot be, however, since $\mathbb{P}^{3}$ is not a Calabi-Yau manifold. There are two issues that complicate the statement that the Landau-Ginzburg is trivial:

1. The Landau-Ginzburg theory is actually a $\mathbb{Z}_{2}$-orbifolded Landau-Ginzburg theory.

2. The Landau-Ginzburg superpotential is not strictly quadric for certain points on a surface $L \subset \mathbb{P}^{3}$.

In [5] it was argued that the proper geometric interpretation for the hybrid phase, at least in the language of the gauged linear $\sigma$-model, is that of a double cover of $\mathbb{P}^{3}$ branched over $L$. Such a space is a (singular) Calabi-Yau threefold. Will our D-brane probes give the same answer?

The analysis of this case in very similar to the bicubic in $\mathbb{P}^{5}$ :

- Define

$$
\mathrm{w}=\frac{S}{\left(x_{0}, \ldots, x_{7}\right)} .
$$

The Koszul resolution of this gives a $128 \times 128$ matrix factorization in analogy with (56).

- Define $\mathrm{O}_{X}$ as the cokernel of the map

$$
S(-2)^{\oplus 4} \stackrel{f_{0}, \ldots, f_{3}}{\longrightarrow} S
$$

The resulting matrix factorization is an $8 \times 8$ factorization which is an analog of 60 . 
- We have monodromy rings:

$$
\begin{aligned}
R_{0}^{\mathbb{Q}} & =\frac{\mathbb{Q}\left[s, s^{-1}\right]}{\kappa(\mathrm{w})} \\
& =\frac{\mathbb{Q}\left[s, s^{-1}\right]}{\left(1-s^{-1}\right)^{8}},
\end{aligned}
$$

and

$$
\begin{aligned}
R_{1}^{\mathbb{Q}} & =\frac{\mathbb{Q}\left[s, s^{-1}\right]}{\kappa\left(\mathrm{O}_{\mathrm{X}}\right)} \\
& =\frac{\mathbb{Q}\left[s, s^{-1}\right]}{\left(1-s^{-2}\right)^{4}} .
\end{aligned}
$$

- We have K-theory modules over the monodromy ring:

$$
\begin{aligned}
M_{0}^{\mathbb{Q}} & =\operatorname{Image}\left(\kappa\left(\mathrm{O}_{\mathbf{x}}\right)\right) \\
& =\operatorname{coker}\left(1-s^{-1}\right)^{4},
\end{aligned}
$$

and

$$
\begin{aligned}
M_{1}^{\mathbb{Q}} & =\operatorname{Image}(\kappa(\mathrm{w})) \\
& =\operatorname{coker}\left(1+s^{-1}\right)^{4} .
\end{aligned}
$$

- We have a sequence of inclusions of $R_{1}^{\mathbb{Q}}$-modules

$$
N_{0} \subset N_{1} \subset N_{2} \subset N_{3} \cong M_{1}^{\mathbb{Q}},
$$

where $N_{i}$ is the image of $\left(1+s^{-1}\right)^{3-i}$ in coker $\left(1+s^{-1}\right)^{4}$. Let $L_{i}$ be a one-dimensional vector subspace of $N_{i}$ such that $N_{i}=N_{i+1} \oplus L_{i}$ (and set $L_{0}=N_{0}$ ). An object in $L_{i}$ has a period that goes as

$$
Z \sim z^{\frac{1}{2}} \log (z)^{i},
$$

as $z \rightarrow 0$ in the hybrid limit.

- The Kähler prepotential is given in the hybrid limit by

$$
e^{-K} \sim|z|(\log |z|)^{3},
$$

yielding the same metric (up to a factor of 3 ) as $(78)$ and so the hybrid limit is again an infinite distance away in the moduli space. We therefore again interpret it as a decompactification. 
Set $B+i J \sim \frac{1}{2 \pi i} \log (z)$ to simulate the Kähler form on this infinite sized space appearing in the hybrid limit and put $\operatorname{Vol}(X) \sim(\log |z|)^{3}$. The masses of objects in $L_{i}$ then go as $J^{i}$ as would befit $2 i$-branes on our new space.

Following the construction of 0 -branes on $\mathbb{P}^{1}$ in section 3.5 we can construct analogous $2 i$-branes on $\mathbb{P}^{3}$ for this hybrid model. Define

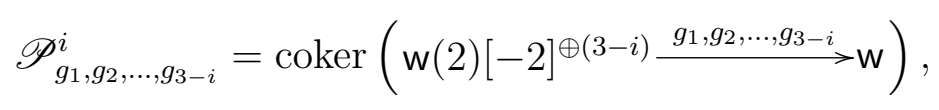

where $g_{1}, g_{2}, \ldots, g_{3-i}$ are linearly independent linear functions in $p_{0}, \ldots, p_{3}$. It follows that $\mathscr{P}_{g_{1}, g_{2}, \ldots, g_{3-i}}^{i}$ is naturally identified with a D-brane wrapping the $2 i$-dimensional subspace of $\mathbb{P}^{3}$ given by $g_{1}=g_{2}=\ldots=g_{3-i}=0$. Note also that the K-theory class of $\mathscr{P}_{g_{1}, g_{2}, \ldots, g_{3-i}}^{i}$ is $\left(1-s^{-2}\right)^{i} \kappa(\mathrm{w})$ and so lies in $L_{i}$. Thus the mass scales as expected for a $2 i$-brane as the $\mathbb{P}^{3}$ becomes large.

For four linearly independent linear functions $g_{1}, \ldots, g_{4}$ one has in the hybrid phase

$$
\operatorname{coker}\left(\mathrm{w}(2)[-2]^{\oplus 4} \stackrel{g_{1}, \ldots, g_{4}}{\longrightarrow} \mathrm{w}\right)=0 .
$$

From this, one can deduce

$$
\text { Cone }\left(\mathscr{P}_{g_{1}, g_{2}, g_{3}}^{0}(2)[-2] \stackrel{f}{\longrightarrow} \mathscr{P}_{g_{1}, g_{2}, g_{3}}^{0}\right)=0
$$

for any $f$ linearly independent to $g_{1}, g_{2}, g_{3}$. This gives an equivalence of 0 -branes at the same point

$$
\mathscr{P}_{g_{1}, g_{2}, g_{3}}^{0}(2) \cong \mathscr{P}_{g_{1}, g_{2}, g_{3}}^{0}[2]
$$

There are therefore two 0-branes, $\mathscr{P}_{g_{1}, g_{2}, g_{3}}^{0}$ and $\mathscr{P}_{g_{1}, g_{2}, g_{3}}^{0}(1)$, for each point in $\mathbb{P}^{3}$.

We further note that these two 0-branes have opposite K-theory classes and so the Dbrane charges of $\mathscr{P}_{g_{1}, g_{2}, g_{3}}^{0}$ and $\mathscr{P}_{g_{1}, g_{2}, g_{3}}^{0}(1)[1]$ coincide. Thus, if one were to try to compute the moduli space of 0-branes in a particular K-theory class it would appear to look a lot like a double cover of $\mathbb{P}^{3}$.

This D-brane interpretation of the geometry of the hybrid limit appears to be getting very close to the proposals of $[5,30]$. There it was proposed that the relevant geometry is a double cover of $\mathbb{P}^{3}$ branched over some surface. The difference we seem to have with this proposal is that we see no branching in the double cover.

The 0-branes $\mathscr{P}_{g_{1}, g_{2}, g_{3}}^{0}$ are $1024 \times 1024$ matrix factorizations of the superpotential $\mathcal{W}$ where the matrix entries are polynomials in $p_{j}$ 's and $x_{i}$ 's. In particular, the matrix factorizations are single-valued functions of $p_{j}$. In going around a loop in $\mathbb{P}^{3}$ it is clearly not the case that a matrix factorization of the form $\mathscr{P}_{g_{1}, g_{2}, g_{3}}^{0}$ can turn into $\mathscr{P}_{g_{1}, g_{2}, g_{3}}^{0}(1)[1]$ in any obvious sense.

Having said that, the geometrical interpretation of $[5,30]$ is very appealing and it would be nice to understand exactly how these apparently different double covers of $\mathbb{P}^{3}$ can be reconciled. The discrepancy may be associated with the fact that our 0-branes $\mathscr{P}_{g_{1}, g_{2}, g_{3}}^{0}$ have a K-theory class which is 2-divisible in the same way that the 0-branes in section 3.5 were 3 -divisible. It would also be interesting to better understand the relationship between the our 0-branes $\mathscr{P}_{g_{1}, g_{2}, g_{3}}^{0}$ and the spinor bundles of [30]. 


\section{Pseudo-Hybrid Limits}

\subsection{A weighted hybrid}

Consider a model with fields $p_{0}, p_{1}, p_{2}, x_{0}, \ldots, x_{6}$ with charges $(-3,-2,-2,1,1, \ldots, 1)$ respectively and a superpotential

$$
\mathcal{W}=p_{0} f_{0}\left(x_{i}\right)+p_{1} f_{1}\left(x_{i}\right)+p_{2} f_{2}\left(x_{i}\right)
$$

where $f_{0}\left(x_{i}\right)$ is a homogeneous polynomial of degree 3 in $x_{0}, \ldots, x_{7}$, and $f_{1}\left(x_{i}\right)$ and $f_{2}\left(x_{i}\right)$ are homogeneous polynomials of degree 2 . For $r \gg 1, B_{\Sigma}=\left(x_{0}, x_{1}, \ldots, x_{6}\right)$ and the low-energy theory is a nonlinear $\sigma$-model on the Calabi-Yau space $X$ given by the intersection $f_{a}=0$ in $\mathbb{P}^{6}$. For sufficiently generic choice of the polynomials this is smooth. The $R$-symmetry assigns charge 2 to $p_{a}$ and leaves $x_{i}$ invariant.

For $r \ll-1$ we find a new kind of hybrid behavior. Here $B_{\Sigma}=\left(p_{0}, p_{1}, p_{2}\right)$. The superpotential terms then constrain classical vacua to $x_{i}=0$ and the space of classical vacua is the weighted projective space $\mathbb{P}_{\{3,2,2\}}^{2}$, with homogeneous coordinates $\left[p_{0}, p_{1}, p_{2}\right]$. Classically this has radius $-r$ and in the limit we have a large base. As we will see, our analysis shows that this classical expectation is not valid.

In generic vacua the gauge symmetry is broken completely. There are two special loci in the vacuum manifold: the point $P=[1,0,0]$ where there is an unbroken $\mathbb{Z}_{3}$, and the curve $C=\left[0, p_{1}, p_{2}\right]$ where there is an unbroken $\mathbb{Z}_{2}$. The $x_{i}$ do not acquire masses from the $D$-terms and interact via a Landau-Ginzburg superpotential with coefficients linear in the homogeneous coordinates on the base. In generic vacua the Landau-Ginzburg superpotential is quadratic, the $x_{i}$ are massive, and the central charge of the Landau-Ginzburg fibre is zero. Related to this is the fact that generic vacua do not preserve our $R$-symmetry. The $R$-symmetry is preserved (up to a gauge transformation) precisely in vacua on the special loci. At the point $P$ the preserved symmetry leaves $p_{0}$ invariant while assigning $R$-charge $2 / 3$ to all the other fields; along the curve $C$ the preserved symmetry (leaving $p_{1}$ and $p_{2}$ invariant) assigns charge 1 to $x_{i}$ and charge -1 to $p_{0}$.

These facts tell us that if we can think of the hybrid model as a fibration, this interpretation cannot be as straightforward as was the case in our previous models. If a fibre-wise approximation is valid, we would expect that generic classical vacua do not lead to superconformal field theories at low energies. The special vacua that do seem to produce such theories lead to theories that are not of the simple type encountered previously.

We now apply the methods we have described to see whether the D-brane structure near the limit can help resolve these puzzles. The homogeneous coordinate ring is

$$
S=\mathbb{C}\left[p_{0}, p_{1}, p_{2}, x_{0}, \ldots, x_{6}\right],
$$

with respective grading $(-3,-2,-2,1,1, \ldots, 1)$. The $R$-charge of each $p_{j}$ is set to 2 while the $x_{i}$ 's are all $R$-charge 0 .

The analysis of this case is once more very similar to the bicubic in $\mathbb{P}^{5}$, at least at first: 
- Define

$$
\mathrm{w}=\frac{S}{\left(x_{0}, \ldots, x_{6}\right)} .
$$

The Koszul resolution of this gives a $64 \times 64$ matrix factorization in analogy with 566 .

- Define $\mathrm{O}_{X}$ as the cokernel of the map

$$
S(-3) \oplus S(-2)^{\oplus 2} \stackrel{f_{0}, f_{1}, f_{2}}{\longrightarrow} S
$$

The resulting matrix factorization is an $4 \times 4$ matrix factorization which is an analog of 60$)$.

- We have monodromy rings:

$$
\begin{aligned}
R_{0}^{\mathbb{Q}} & =\frac{\mathbb{Q}\left[s, s^{-1}\right]}{\kappa(\mathrm{w})} \\
& =\frac{\mathbb{Q}\left[s, s^{-1}\right]}{\left(1-s^{-1}\right)^{7}},
\end{aligned}
$$

and

$$
\begin{aligned}
R_{1}^{\mathbb{Q}} & =\frac{\mathbb{Q}\left[s, s^{-1}\right]}{\kappa\left(\mathrm{O}_{\mathbf{X}}\right)} \\
& =\frac{\mathbb{Q}\left[s, s^{-1}\right]}{\left(1-s^{-3}\right)\left(1-s^{-2}\right)^{2}} .
\end{aligned}
$$

- We have K-theory modules over the monodromy ring:

$$
\begin{aligned}
M_{0}^{\mathbb{Q}} & =\operatorname{Image}\left(\kappa\left(\mathrm{O}_{\mathbf{x}}\right)\right) \\
& =\operatorname{coker}\left(1-s^{-1}\right)^{4},
\end{aligned}
$$

and

$$
\begin{aligned}
M_{1}^{\mathbb{Q}} & =\operatorname{Image}(\kappa(\mathrm{w})) \\
& =\operatorname{coker}\left(1+s^{-1}+s^{-2}\right)\left(1+s^{-1}\right)^{2} .
\end{aligned}
$$

The form of (118) makes this case physically very different to the true hybrids considered above. First consider

$$
P=\text { Image }\left(1+s^{-1}\right)^{2} \subset M_{1}^{\mathbb{Q}} .
$$

This is a two dimensional submodule with monodromy of order three around the limit $z=0$. Thus we have two periods with a limiting behavior given by $z^{\frac{1}{3}}$. Next we have

$$
N_{0} \subset N_{1} \subset M_{1}^{\mathbb{Q}},
$$


where $N_{i}=$ Image $\left(1+s^{-1}+s^{-2}\right)\left(1+s^{-1}\right)^{1-i}$. As above define $L_{0}=N_{0}$ and $L_{1}$ such that $N_{1}=L_{0} \oplus L_{1}$. Periods associated to $L_{0}$ and $L_{1}$ must go as $z^{\frac{1}{2}}$ and $z^{\frac{1}{2}} \log (z)$ respectively. We also have $M_{1}^{\mathbb{Q}}=P \oplus L_{0} \oplus L_{1}$.

Computing the symplectic form yields a Kähler potential of the form

$$
e^{-K} \sim|z|^{\frac{2}{3}}+|z| \log |z|+\ldots
$$

This gives a metric on the moduli space

$$
g_{z \bar{z}} \sim-|z|^{-\frac{5}{3}} \log |z|+\ldots
$$

which puts the origin $z=0$ at a finite distance away from a generic point in the moduli space. This limit is not associated to a decompactification of the target space.

Since we do not have a decompactification, we do not need to rescale the D-brane masses by any volume factor to understand their behaviour near $z=0$. Accordingly we compute $e^{K / 2}|Z|$ to see the D-brane masses.

It follows that the D-branes associated to $N$ have masses that are nonzero and finite as $z \rightarrow 0$ while those associated to $L_{0}$ and $L_{1}$ have masses that go to zero. Such massless D-branes are indicative of a singular conformal field theory [31]. Indeed, one can compute the normalized Yukawa coupling as in [6] and it diverges.

\subsection{Explicit D-branes}

Let us try to explicitly construct D-branes that are associated with charges in $P, L_{0}$ and $L_{1}$.

Copying previous constructions we set

$$
\begin{aligned}
\mathscr{P}_{\left[0, x_{1}, x_{2}\right]}^{0} & =\operatorname{coker}\left(\mathrm{w}(3)[-2] \oplus \mathrm{w}(2)[-2] \stackrel{\left(p_{0} x_{2} p_{1}-x_{1} p_{2}\right)}{\longrightarrow} \mathrm{w}\right) \\
\mathscr{P}_{[1,0,0]}^{0} & =\operatorname{coker}\left(\mathrm{w}(2)[-2]^{\oplus 2} \stackrel{\left(p_{1} p_{2}\right)}{\longrightarrow} \mathrm{w}\right) .
\end{aligned}
$$

The objects $\mathscr{P}_{\left[0, x_{1}, x_{2}\right]}^{0}$ are 0 -branes living on the curve $C$ where we define $C \subset \mathbb{P}_{\{3,2,2\}}^{2}$ by setting $p_{0}=0$. These have charge in class $L_{0}$ and so all become massless as $z \rightarrow 0$.

The object $\mathscr{P}_{[1,0,0]}^{0}$ is a 0 -brane stuck at the point $[1,0,0]$. It is in the class $P$ and so has nonzero mass in the hybrid limit.

The new feature arising from the fact that the base in the hybrid is a weighted projective space is that there is no clear construction of a 0-brane that lives at any point other than on $C$ or $[1,0,0]$. Classically if one were to write a free resolution of skyscraper sheaf at an arbitrary point on $\mathbb{P}_{\{3,2,2\}}^{2}$ then one would try something like

$$
\mathscr{O}_{x}=\operatorname{coker}\left(\mathscr{O}(-6)^{\oplus 2} \stackrel{\left(f_{0}, f_{1}\right)}{\longrightarrow} \mathscr{O}\right) \text {, }
$$

where $f_{i}$ are of the form $a_{0} x_{0}^{2}+a_{1} x_{1}^{3}+a_{2} x_{2}^{3}$ for complex $a_{0}, a_{1}, a_{2}$. In the case of the hybrid model we cannot form combinations like $a_{0} p_{0}^{2}+a_{1} p_{1}^{3}+a_{2} p_{2}^{3}$ because they violate homogeneity of the $R$-charge grading. 


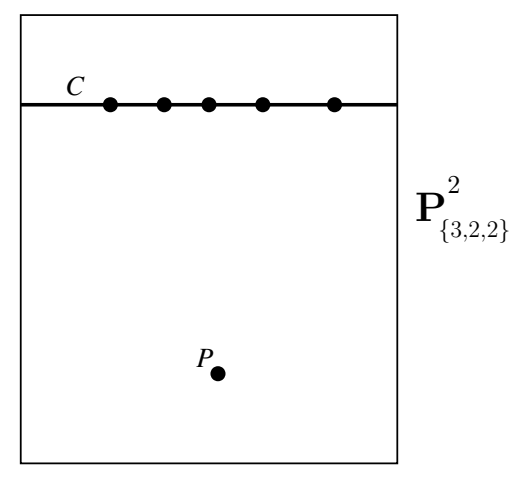

Figure 2: Apparent Target Space.

We may also construct

$$
\mathscr{P}_{C}^{1}=\operatorname{coker}\left(\mathrm{w}(3)[-2] \stackrel{p_{0}}{\longrightarrow} \mathrm{w}\right)
$$

which is naturally associated to the 2-brane wrapping the curve $C$. Its class is in $L_{1}$ and so it also becomes massless in the hybrid limit.

We have the rather peculiar state of affairs that the 0-brane probe picture gives something along the lines of figure 2. There is one class of "points" that live on the curve $C$ and only one other isolated point $P$.

\subsection{Spacetime physics at the limit point}

What is the physics associated with the hybrid limit for this model? The set of D-branes that acquires zero mass in this limit corresponds to a whole $\mathbb{P}^{1}$ 's worth of 0 -branes living at point in $C$, the 2-brane wrapping $C$ itself and any stable D-brane whose charge is a linear combination of these.

To determine the true spectrum of massless particles we need to quantize these classical BPS objects. In [32] it was argued that a family of classical D-branes parametrized by $\mathbb{P}^{1}$ gives rise to a massless vector in the uncompactified four-dimensional spacetime. Thus we appear to have an enhanced gauge symmetry. Since there is only one class of 0-branes going massless, the associated gauge symmetry should be $\mathrm{SU}(2)$.

It is interesting to compare this situation to the more conventional picture of nonperturbatively enhanced gauge symmetry appearing in Calabi-Yau compactifications [33, 34]. There one has

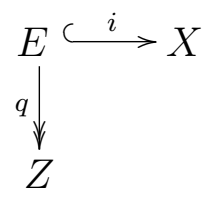

where $E$ is a divisor and $Z$ is a curve which we take to be $\mathbb{P}^{1}$. The enhanced gauge symmetry occurs when $E$ shrinks down to the curve $Z$. In this case it is the family of 2-branes wrapping 
the fibres of the map $q$ which become massless. Note also that the 4-brane wrapping $E$ also becomes massless and this plays the role of the magnetic monopole in the $N=2 \mathrm{SU}(2)$ theory of [35].

In $[36,37]$ it was argued that the spectrum of massless D-branes in $(126)$ is given by (the stable objects in) $i_{*} q^{*} \mathbf{D}(Z)$. That is, it is essentially the derived category of $Z$ mapped into $\mathrm{D}(X)$.

Our hybrid model is similar but geometrically quite distinct. We have points on $C$ and $C$ itself becoming massless. This generates the whole derived category $\mathbf{D}(C)$. Thus, again, it is the derived category of $\mathbb{P}^{1}$ which is associated with an enhanced gauge symmetry. But in our case it is the $\mathbb{P}^{1}$ itself which "shrinks down" and acquires massless wrapped D-branes.

It should also be noted that the theory of enhanced $\mathrm{SU}(2)$ we obtain is no ordinary $\mathrm{SU}(2)$ theory. The Jordan canonical form of the monodromy matrix around the hybrid limit coming from 118 is

$$
\left(\begin{array}{lllr}
\omega & & & \\
& \omega^{2} & & \\
& & -1 & 1 \\
& & & -1
\end{array}\right) .
$$

The lower block is recognizable as the classical monodromy for electric and magnetic charges as observed in $\mathrm{SU}(2)$ theories in [35]. But the monodromy is nontrivial on the other two $\mathrm{RR}$ charges and these are associated to the graviphoton. Thus the SU(2) enhanced gauge symmetry is somehow inextricably intertwined with gravity and is not, therefore, the usual enhanced $\mathrm{SU}(2)$ theory.

Indeed, there is no direction in the moduli space to move where one might try to "engineer" the quantum Seiberg-Witten theory along the lines of [38]. The gauge coupling (or mass scale $\Lambda$ ) of the $\mathrm{SU}(2)$ theory is tied to the gravitation coupling and it is impossible to find any limit where gravity is decoupled from this $\mathrm{SU}(2)$ theory.

\section{Discussion}

Gauged linear $\sigma$-models have been a fruitful tool in studying many aspects of $N=2$ superconformal field theories and the associated string models. The gauge theory description is expected to be a reliable guide to the low-energy physics in the large- $|r|$ limit. We expect that in a general model, most of these limits correspond to some kind of hybrid model.

In a true hybrid phase we have found that the low-energy dynamics can usefully be thought of in terms of a fibration. The fibre is a conformal field theory of charge $\hat{c}<3$ and the parameters of this vary slowly over a large base space of dimension $3-\hat{c}$. For the examples we discuss in the paper the fibre can be thought of as a Calabi-Yau space of dimension $\hat{c}$ that is "stuck" at small size. We have given examples of this for $\hat{c}=2$ (a K3 with Picard number zero) and $\hat{c}=0$ (two points). The instanton expansion in the gauge theory corresponds to an expansion in worldsheet instantons wrapping curves in the base (the fibre theory does not admit field configurations that could contribute to this expansion) 
and the limit in which these can be neglected is the limit in which the base decompactifies. The analysis of finding light "0-branes" reinforces this picture in general.

It is clear, moreover, that true hybrid limits require a special alignment of the charges in the gauge theory. In the examples we have considered the key is that the base $\mathbb{P}^{n}$ is unweighted in the true hybrid case and weighted in the pseudo-hybrid case. For example, an intersection of a quadric and a quartic in $\mathbb{P}^{5}$ (which was not discussed above) leads to another pseudo-hybrid phase, since the base is a weighted $\mathbb{P}^{1}$.

We therefore expect that "most" limit points will in fact be pseudo-hybrid phases. In these phases we have not found a simple description of the limiting behavior when instanton effects can be neglected. The naïve expectation that the fields describing motion on the space of classical vacua are weakly interacting is not valid. Interactions with the other massless fields lead to large corrections to the kinetic terms and the space of classical vacua does not grow large in the limit. Correspondingly, the limit point is at a finite distance in the moduli space and corresponds to a singular conformal field theory.

Since the work of Strominger [31] such singularities have been understood in string theory as associated to the existence of light D-brane states which become massless in the limit. If we can approach the singularity while keeping the volume of the target space large, we can incorporate these modes into an effective theory while decoupling gravitational interactions. This involves taking a limit in which the string coupling $g \rightarrow 0$ and $\alpha^{\prime} \rightarrow 0$ as $z \rightarrow 0$ where $z$ is a local coordinate on the moduli space, in such a way that the effective coupling of the four-dimensional theory remains finite [39]. In the model of section 4 there is no way to decouple gravity from the light modes at the singularity, because in the limit $z \rightarrow 0$ one necessarily shrinks down the whole Calabi-Yau threefold. In the same way, the singularity cannot be seen in 5 dimensions from M-theory compactified on the Calabi-Yau threefold. This is because one must essentially normalize the volume of the threefold to be one when constructing the M-theory moduli space of vector multiplet deformations [32]. This normalization freezes out the deformation we require to reach the singularity.

Obviously further examples will come from considering cases where the moduli space is dimension greater than one. The task of analyzing periods and doing analytic continuation in multiparameter examples (such as [28]) can be formidable but the methods developed in section 2 should simplify this considerably.

Finally, there are many interesting computations that can be performed on our candidate 0-branes which we have not done. First, analysis of stability was started in [24] but this should be investigated further. Secondly, there are basic questions about the moduli space of these 0-branes which remain to be explored. For example, $\operatorname{Ext}^{1}(\mathscr{P}, \mathscr{P})$ represents first-order deformations of D-branes $\mathscr{P}$ and so gives a naïve dimension for the moduli space. One can then test the unobstructedness of such deformations by using the $A_{\infty}$-structure. This gives the true dimension of the moduli space and shows any "fatness" of the moduli space that one might associated with Landau-Ginzburg theories. Such computations seem formidable, at first sight, but would be very interesting. 


\section{Acknowledgments}

We wish to thank I. Melnikov and A. Roy for useful discussions. The authors are supported by an NSF grant DMS-0606578.

\section{References}

[1] E. Witten, Phases of $N=2$ Theories in Two Dimensions, Nucl. Phys. B403 (1993) 159-222, hep-th/9301042.

[2] P. S. Aspinwall, B. R. Greene, and D. R. Morrison, Multiple Mirror Manifolds and Topology Change in String Theory, Phys. Lett. 303B (1993) 249-259.

[3] M. Herbst, K. Hori, and D. Page, Phases Of $N=2$ Theories In $1+1$ Dimensions With Boundary, arXiv:0803.2045.

[4] P. S. Aspinwall and B. R. Greene, On the Geometric Interpretation of $N=2$ Superconformal Theories, Nucl. Phys. B437 (1995) 205-230, hep-th/9409110.

[5] A. Caldararu et al., Non-Birational Twisted Derived Equivalences in Abelian GLSMs, arXiv:0709.3855.

[6] P. Candelas, X. C. de la Ossa, P. S. Green, and L. Parkes, A Pair of Calabi-Yau Manifolds as an Exactly Soluble Superconformal Theory, Nucl. Phys. B359 (1991) $21-74$.

[7] P. S. Aspinwall, D-Branes on Toric Calabi-Yau Varieties, arXiv:0806.2612.

[8] D. A. Cox, The Homogeneous Coordinate Ring of a Toric Variety, J. Algebraic Geom. 4 (1995) 17-50, alg-geom/9210008.

[9] A. Bondal and D. Orlov, Semiorthogonal Decomposition for Algebraic Varieties, alggeom/9506012.

[10] P. S. Aspinwall, D-Branes on Calabi-Yau Manifolds, in J. M. Maldacena, editor, "Progress in String Theory. TASI 2003 Lecture Notes", pages 1-152, World Scientific, 2005, hep-th/0403166.

[11] D. Orlov, Derived Categories of Coherent Sheaves and Triangulated Categories of Singularities, math.AG/0503632.

[12] P. S. Aspinwall, Topological D-Branes and Commutative Algebra, hep-th/0703279, submitted to Communications in Number Theory and Physics.

[13] D. Eisenbud, Homological Algebra on a Complete Intersection, with an Application to Group Representations, Trans. Amer. Math. Soc. 260 (1980) 35-64. 
[14] L. L. Avramov and D. R. Grayson, Resulutions and Cohomology over Complete Intersections, in D. Eisenbud et al., editors, "Computations in Algebraic Geometry with Macaulay 2", Algorithms and Computations in Mathematics 8, pages 131-178, Springer-Verlag, 2001.

[15] T. H. Gulliksen, A Change of Ring Theorem with Applications to Poincare Series and and Intersection Multiplicity, Math. Scand. 34 (1974) 167-183.

[16] D. R. Morrison, Mirror Symmetry and Rational Curves on Quintic Threefolds: A Guide For Mathematicians, J. Amer. Math. Soc. 6 (1993) 223-247, alg-geom/9202004.

[17] P. S. Aspinwall, The Landau-Ginzburg to Calabi-Yau Dictionary for D-Branes, J. Math. Phys. 48 (2007) 082304, hep-th/0610209.

[18] A. Libgober and J. Teitelbaum, Lines on Calabi-Yau Complete Intersections, Mirror Symmetry, and Picard-Fuchs Equations., Internat. Math. Res. Notices 1993, no. 1, 29-39. (1993) 29-39.

[19] N. Seiberg, Observations on the Moduli Space of Superconformal Field Theories, Nucl. Phys. B303 (1988) 286-304.

[20] C. Vafa, Superstring Vacua, Presented at Symp. on Fields, Strings and Quantum Gravity, Beijing, China, May 29 - Jun 10, 1989.

[21] P. Candelas, E. Derrick, and L. Parkes, Generalized Calabi-Yau Manifolds and the Mirror of a Rigid Manifold, Nucl. Phys. B407 (1993) 115-154.

[22] S. Sethi, Supermanifolds, Rigid Manifolds and Mirror Symmetry, Nucl. Phys. B430 (1994) 31-50, hep-th/9404186.

[23] P. S. Aspinwall, K3 Surfaces and String Duality, in C. Efthimiou and B. Greene, editors, "Fields, Strings and Duality, TASI 1996", pages 421-540, World Scientific, 1997, hep-th/9611137.

[24] P. S. Aspinwall, Probing Geometry with Stability Conditions, arXiv:0905.3137.

[25] D. S. Dummit and R. M. Foote, Abstract Algebra, Prentice Hall, 2003.

[26] P. Candelas and X. C. de la Ossa, Moduli Space of Calabi-Yau Manifolds, Nucl. Phys. B355 (1991) 455-481.

[27] P. S. Aspinwall, B. R. Greene, and D. R. Morrison, Measuring Small Distances in $N=2$ Sigma Models, Nucl. Phys. B420 (1994) 184-242, hep-th/9311042.

[28] P. S. Aspinwall, Minimum Distances in Non-Trivial String Target Spaces, Nucl. Phys. B431 (1994) 78-96, hep-th/9404060. 
[29] M. R. Douglas, D-Branes, Categories and N=1 Supersymmetry, J. Math. Phys. 42 (2001) 2818-2843, hep-th/0011017.

[30] N. Addington, The Derived Category of the Intersection of Four Quadrics, arXiv: 0904.1764.

[31] A. Strominger, Massless Black Holes and Conifolds in String Theory, Nucl. Phys. B451 (1995) 96-108, hep-th/9504090.

[32] E. Witten, Phase Transitions in M-Theory and F-Theory, Nucl. Phys. B471 (1996) 195-216, hep-th/9603150.

[33] P. S. Aspinwall, Enhanced Gauge Symmetries and Calabi-Yau Threefolds, Phys. Lett. B371 (1996) 231-237, hep-th/9511171.

[34] S. Katz, D. R. Morrison, and M. R. Plesser, Enhanced Gauge Symmetry in Type II String Theory, Nucl. Phys. B477 (1996) 105-140, hep-th/9601108.

[35] N. Seiberg and E. Witten, Electric - Magnetic Duality, Monopole Condensation, and Confinement in N=2 Supersymmetric Yang-Mills Theory, Nucl. Phys. B426 (1994) 19-52, hep-th/9407087, (erratum-ibid. B430 (1994) 485-486).

[36] R. P. Horja, Derived Category Automorphisms from Mirror Symmetry, math.AG/0103231.

[37] P. S. Aspinwall, R. L. Karp, and R. P. Horja, Massless D-branes on Calabi-Yau threefolds and monodromy, Commun. Math. Phys. 259 (2005) 45-69, hep-th/0209161.

[38] S. Kachru and C. Vafa, Exact Results For N=2 Compactifications of Heterotic Strings, Nucl. Phys. B450 (1995) 69-89, hep-th/9505105.

[39] S. Kachru et al., Nonperturbative Results on the Point Particle Limit of N=2 Heterotic String Compactifications, Nucl. Phys. B459 (1996) 537-558, hep-th/9508155. 